Supporting Information

\title{
A Density Functional Investigation of the Mitsunobu Reaction
}

\author{
Stephan Schenk, Jennie Weston, and Ernst Anders*
}

\section{Contents}

1 Full Author Listing for References 31 and $32 \quad$ S2

2 Carbonyl Subtituted Diazenes S3

2.1 Results . . . . . . . . . . . . . . . . . . . . . S3

2.2 Computational Details $\ldots \ldots \ldots \ldots \ldots \ldots \ldots$. . . . . . . . . . .

3 Table of Electronic Properties $\quad$ S6

4 Absolute Energies of Calculated Structures $\quad$ S7

5 Cartesian Coordinates of Calculated Structures $\quad$ S10

5.1 Coordinates in Solution . . . . . . . . . . . . . . . . S10

5.2 Coordinates in the Gas Phase . . . . . . . . . . . . . . . . S34

6 Effect of Substitution on Phosphorus $\quad$ S58

6.1 Absolute Energies of Calculated Structures . . . . . . . . . . . . . . . . . S58

6.2 Cartesian Coordinates of Calculated Structures . . . . . . . . . . . . . . . S59 


\section{Full Author Listing for References 31 and 32}

(31) Frisch, M. J.; Trucks, G. W.; Schlegel, H. B.; Scuseria, G. E.; Robb, M. A.; Cheeseman, J. R.; Zakrzewski, V. G.; Montgomery, Jr., J. A.; Stratmann, R. E.; Burant, J. C.; Dapprich, S.; Millam, J. M.; Daniels, A. D.; Kudin, K. N.; Strain, M. C.; Farkas, O.; Tomasi, J.; Barone, V.; Cossi, M.; Cammi, R.; Mennucci, B.; Pomelli, C.; Adamo, C.; Clifford, S.; Ochterski, J.; Petersson, G. A.; Ayala, P. Y.; Cui, Q.; Morokuma, K.; Rega, N.; Salvador, P.; Dannenberg, J. J.; Malick, D. K.; Rabuck, A. D.; Raghavachari, K.; Foresman, J. B.; Cioslowski, J.; Ortiz, J. V.; Baboul, A. G.; Stefanov, B. B.; Liu, G.; Liashenko, A.; Piskorz, P.; Komaromi, I.; Gomperts, R.; Martin, R. L.; Fox, D. J.; Keith, T.; Al-Laham, M. A.; Peng, C. Y.; Nanayakkara, A.; Challacombe, M.; Gill, P. M. W.; Johnson, B.; Chen, W.; Wong, M. W.; Andres, J. L.; Gonzalez, C.; HeadGordon, M.; Replogle, E. S.; Pople, J. A. "Gaussian98, Revision A.11-4”, 2002 Gaussian, Inc., Pittsburgh PA.

(32) Frisch, M. J.; Trucks, G. W.; Schlegel, H. B.; Scuseria, G. E.; Robb, M. A.; Cheeseman, J. R.; J. A. Montgomery, Jr., J. A.; Vreven, T.; Kudin, K. N.; Burant, J. C.; Millam, J. M.; Iyengar, S. S.; Tomasi, J.; Barone, V.; Mennucci, B.; Cossi, M.; Scalmani, G.; Rega, N.; Petersson, G. A.; Nakatsuji, H.; Hada, M.; Ehara, M.; Toyota, K.; Fukuda, R.; Hasegawa, J.; Ishida, M.; Nakajima, T.; Honda, Y.; Kitao, O.; Nakai, H.; Klene, M.; Li, X.; Knox, J. E.; Hratchian, H. P.; Cross, J. B.; Adamo, C.; Jaramillo, J.; Gomperts, R.; Stratmann, R. E.; Yazyev, O.; Austin, A. J.; Cammi, R.; Pomelli, C.; Ochterski, J. W.; Ayala, P. Y.; Morokuma, K.; Voth, G. A.; Salvador, P.; Dannenberg, J. J.; Zakrzewski, V. G.; Dapprich, S.; Daniels, A. D.; Strain, M. C.; Farkas, O.; Malick, D. K.; Rabuck, A. D.; Raghavachari, K.; Foresman, J. B.; Ortiz, J. V.; Cui, Q.; Baboul, A. G.; Clifford, S.; Cioslowski, J.; Stefanov, B. B.; Liu, G.; Liashenko, A.; Piskorz, P.; Komaromi, I.; Martin, R. L.; Fox, D. J.; Keith, T.; Al-Laham, M. A.; Peng, C. Y.; Nanayakkara, A.; Challacombe, M.; Gill, P. M. W.; Johnson, B.; Chen, W.; Wong, M. W.; Gonzalez, C.; Pople, J. A. "Gaussian03, Revision C.02", 2004 Gaussian, Inc., Wallingford CT. 


\section{Carbonyl Subtituted Diazenes}

\subsection{Results}

Before addressing the mechanism of the Mitsunobu reaction, we first assessed the energetical and structural properties of a series of four carbonyl substituted diazenes in a general manner. For the two simplest diazenes (formyldiazene $\mathbf{2 a}$ and acetyldiazene $\mathbf{2 b}$ ), six different conformers are theoretically possible - four planar and two nonplanar ones (Chart S1). An extended search of the conformational hypersurface demonstrated that the cis $c$ conformers of both diazenes are not stationary points. The remaining conformers are either energetically stable structures or, when steric interactions are present, transition structures for rotation about the $\mathrm{C}-\mathrm{N}$ bond. The planar trans a conformer is the global minimum for $\mathbf{2 a}$ whereas the nonplanar trans $c$ is the most stable structure for $\mathbf{2 b}$ (see Table $\mathrm{S} 1$ for their relative stabilities).

As the flexibility of the diazene grows [diformyldiazene $\mathbf{2 c}$ and dimethylazodicarboxylate $\mathbf{2 d}$ (DMAD)], the number of possible conformers increases accordingly. Only the energetically stable conformers of the latter two diazenes are given in Table S1. The conformers of interest are named according to Chart $\mathrm{S} 1$ with footnotes in the table defining the additional dihedral angles necessary to unambiguously define the three dimensional structure of the conformer. The same general structural pattern calculated for $\mathbf{2 b}$ is also observed for $\mathbf{2 c}$ and $\mathbf{2 d}$. As steric hindrance increases, the planar $a$ and $b$ conformers become transition structures for rotation about the $\mathrm{C}-\mathrm{N}$ bonds. In the case of $\mathbf{2 d}$, only nonplanar $c / c$ conformers are energetical minima.

Optimization of the different conformers of $\mathbf{2 a - 2 d}$ in acetonitrile (Table S1) showed that the relative energies of the different conformers do not change significantly upon solvation. For all diazenes, a fast equilibrium between several different conformers is to be expected in solution. When bulkier substituents are present ( $\mathbf{2 b}$ and $\mathbf{2 d}$ ), there is a clear preference for a single trans conformation which is illustrated in Chart $\mathrm{S} 2$ for the experimentally relevant DMAD (2d). For the calculations performed in this article, only the most stable conformation of $\mathbf{2} \mathbf{d}$ was considered further.
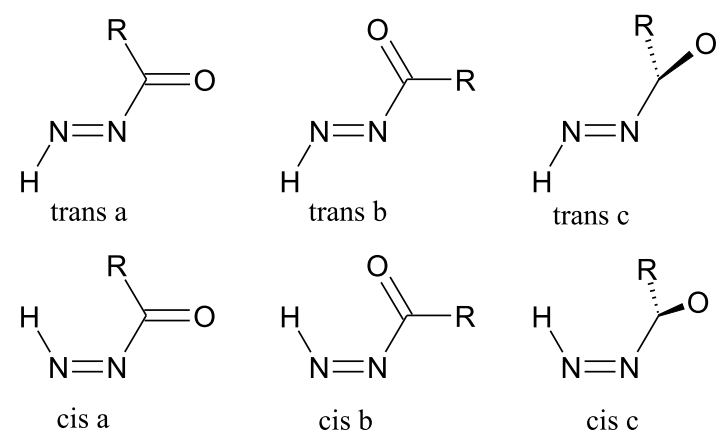

Chart S1: The six different possible conformations of the monsubstituted diazenes $\mathbf{2 a}(\mathrm{R}=\mathrm{H})$ and $\mathbf{2 b}\left(\mathrm{R}=\mathrm{CH}_{3}\right)$. 


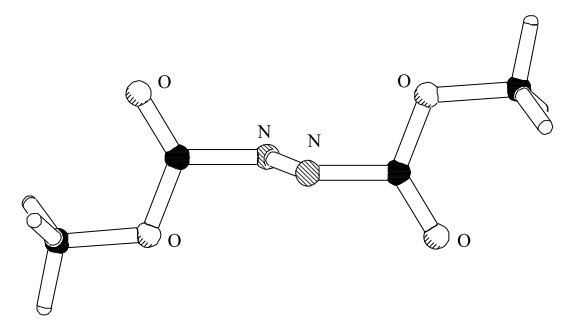

Chart S2: The most stable conformation of DMAD (2d).

The global minimum of all four carbonyl substituted diazenes is a trans conformation. This is in direct contrast to halogen substituted diazenes which prefer a cis conformation. Investigations on $\mathrm{X}^{1} \mathrm{~N}=\mathrm{NX}^{2}$ diazenes ${ }^{\mathrm{S} 1}\left(\mathrm{X}^{1,2}=\right.$ halogen or hydrogen) have shown that this cis preference can be interpreted as the sum of three different electronic interactions: (1) negative hyperconjugation. Partial delocalization of the lone pair on nitrogen (N1) into the $\sigma_{N 2-X}^{*}$ orbital leads to a significant stabilization of the cis form. (2) Electrostatic interactions between the two substituents $\mathrm{X}^{1}$ and $\mathrm{X}^{2}$. This stabilizes the cis form of unsymmetrical diazenes $\left(X^{1}=H ; X^{2}=\right.$ halogen $)$ and destabilizes that of symmetric diazenes. (3) Electrostatic interactions between the lone electron pairs on adjacent $\mathrm{N}$ atoms which destabilizes the cis conformer. In diazene itself $(\mathrm{HN}=\mathrm{NH})$, the hyperconjugative interactions are weak, leading to the domination of the electrostatic effects (2) and (3) thus resulting in a trans preference. ${ }^{\mathrm{S} 1}$

As with diazene, the hyperconjugative interactions, especially the $\pi_{N=N} \rightarrow \pi_{C=O}^{*}$ and the $n_{N} \rightarrow \sigma_{C-N}^{*}$ interactions in the carbonyl substituted derivatives $\mathbf{2 a - 2 d}$, are relatively small $\left(<10 \mathrm{kcal} \mathrm{mol}^{-1}\right)$. The trans preference is clearly determined by steric and electrostatic effects which increase as the size/number of substituents increases. The preference for a nonplanar conformation is also steric in origin due to strong electrostatic repulsions between the substituent on the carbonyl group(s) and the lone pair orbitals on the nitrogen atoms.

The successive replacement of $\mathrm{H}$ in diazene itself with carbonyl groups has quite some influence upon the electrophilicity of the diazene. The negative charge on nitrogen $\left(q_{N}\right)$ is reduced considerably upon substitution (Table S2) thus facilitating the initial approach of a nucleophile. Carbonyl substitution also influences the $\pi_{N=N}^{*}$ orbital in a positive way since its energy is considerably lowered, i. e. the orbital is stabilized upon substitution. This increases the ability of this orbital to interact with a lone electron pair orbital on an incoming nucleophile such as triphenylphosphine.

\subsection{Computational Details}

As we have reported in the past, low level theoretical methods (semiempirical and HF methods) are not capable of properly describing the relative energies of the different conformers of simple diazenes. ${ }^{\text {S1 }}$ A correlated method combined with a large basis set has to be employed in order

(S1) Nordhoff, K.; Anders, E. J. Org. Chem. 1999, 64, 7485-7491. 
Table S1: Relativ Gibbs' free enthalpies $\left(\mathrm{kcal} \mathrm{mol}^{-1}\right)$ of the different conformers of carbonyl substituted diazenes in the gas phase $\left(\Delta G_{\text {gas }}\right)$ and in acetonitrile $\left(\Delta G_{\text {soln. }}\right)$.

\begin{tabular}{|c|c|c|c|c|c|}
\hline conformer & $\Delta G_{\text {gas }}$ & $\Delta G_{\text {soln }}$ & conformer & $\Delta G_{\text {gas }}$ & $\Delta G_{\text {soln }}$ \\
\hline \multicolumn{3}{|c|}{ formyldiazene $\mathbf{2 a}$} & \multicolumn{3}{|c|}{ acetyldiazene $\mathbf{2 b}$} \\
\hline cis a & $2.8^{a}$ & $3.6^{a}$ & cis $a$ & $7.5^{a}$ & $6.7^{a}$ \\
\hline cis $b$ & 2.4 & 2.0 & $c i s b$ & 0.6 & 1.7 \\
\hline trans a & 0.0 & 0.0 & trans $a$ & $2.1^{a}$ & 1.9 \\
\hline trans $b$ & $4.8^{a}$ & $4.6^{a}$ & $\operatorname{trans} b$ & $3.9^{a}$ & 3.6 \\
\hline $\operatorname{trans} c$ & 1.0 & 0.3 & $\operatorname{trans} c$ & 0.0 & 0.0 \\
\hline \multicolumn{3}{|c|}{ diformyldiazene $\mathbf{2} \mathbf{c}^{b}$} & \multicolumn{3}{|c|}{ DMAD $\mathbf{2} \mathbf{d}^{b}$} \\
\hline $\operatorname{cis} c / c^{c}$ & 3.2 & 3.1 & $\operatorname{cis} c / c^{c, d}$ & 9.2 & 7.3 \\
\hline $\operatorname{trans} c / c^{e}$ & 1.6 & 1.2 & $\operatorname{cis} c / c^{c, f}$ & 3.6 & 4.3 \\
\hline $\operatorname{trans} c / c^{c}$ & 1.0 & 0.7 & trans $c / c^{c, g}$ & 7.9 & 8.1 \\
\hline $\operatorname{trans} b / c^{h}$ & 1.0 & 0.5 & trans $c / c^{c, g}$ & 4.8 & 4.4 \\
\hline $\operatorname{trans} b / c^{i}$ & 0.3 & 0.0 & trans $c / c^{c, d}$ & 4.2 & 4.3 \\
\hline $\operatorname{trans} a / a^{j}$ & 0.0 & 0.3 & trans $c / c^{c, g}$ & 0.0 & 0.0 \\
\hline
\end{tabular}

\footnotetext{
First order transition structure for rotation about the $\mathrm{C}-\mathrm{N}$ bond.

Only energetically stable conformers for $\mathbf{2 c}$ and $\mathbf{2 d}$ are given.

$\angle \mathrm{OCNN}=90^{\circ} ;-90^{\circ}$

$\angle \mathrm{MeOCN}=0^{\circ} ; 0^{\circ}$

$\angle \mathrm{OCNN}=90^{\circ} ; 90^{\circ}$

$\angle \mathrm{MeOCN}=0^{\circ} ; 180^{\circ}$

$\angle \mathrm{MeOCN}=180^{\circ} ; 180^{\circ}$

$\angle \mathrm{OCNN}=90^{\circ} ; 180^{\circ}$

$\angle \mathrm{OCNN}=90^{\circ} ; 0^{\circ}$

$\angle \mathrm{OCNN}=180^{\circ} ; 180^{\circ}$
}

Table S2: Selected electronic properties of the most stable conformer of the substituted diazenes $\mathbf{2 a}-\mathbf{2 d}$ as compared to $\mathrm{HN}=\mathrm{NH}$.

\begin{tabular}{cccccc}
\hline & diazene & 2a & 2b & 2c & 2d \\
\hline$q_{N 1}{ }^{a}$ & -0.295 & -0.213 & -0.185 & -0.168 & -0.171 \\
$q_{N 2}{ }^{a}$ & -0.295 & -0.276 & -0.286 & -0.168 & -0.171 \\
$\varepsilon\left(\pi_{N=N}^{*}\right)^{b}$ & +12.1 & -2.6 & -2.5 & -2.7 & -2.4 \\
\hline
\end{tabular}

$a \quad$ Atomic charge (NBO analysis).

$b \quad$ Energy $(\mathrm{eV})$ of the $\pi_{N=N}^{*}$ orbital. 
to describe these compounds correctly with the best theoretical results being obtained using the $\mathrm{BP}^{\mathrm{S} 2, \mathrm{~S} 3}$ density functional with the very large $6-311++\mathrm{G}(3 \mathrm{df}, 3 \mathrm{pd})^{\mathrm{S} 4}$ basis set. ${ }^{\mathrm{S} 1}$ Since this level

of theory is rather computationally demanding, especially when bulky substituents are present, we performed an extended conformational search at the HF/6-311+G(d,p) level for the series of diazenes considered here. The HF structures then served as starting geometries for full geometry optimizations and frequency calculations for all intermediates at the BP86/6-311++G(3df,3pd) level of theory.

\section{Table of Electronic Properties}

Table S3: Selected electronic (NBO) properties of the compounds involved in the diazene/phosphine reaction. Calculated at the BP86/6-311++G(3df,3pd) level of theory.

\begin{tabular}{|c|c|c|c|c|c|c|c|}
\hline & $q_{P}^{a}$ & $q_{N 1}{ }^{a}$ & $q_{N 2}{ }^{a}$ & $q_{O^{a}}^{a}$ & $\varepsilon_{N 1}^{b}$ & $\varepsilon_{N 2}^{b}$ & $\overline{\varepsilon_{O} b}$ \\
\hline $\mathrm{PH}_{3}$ & 0.00 & & & & & & \\
\hline $3 d$ & +0.99 & -0.58 & -0.49 & -0.66 & $c$ & -9.5 & -7.4 \\
\hline $14 b$ & +1.09 & -0.60 & -0.42 & -0.74 & -7.0 & -10.1 & -7.5 \\
\hline 2d & & -0.17 & -0.17 & -0.49 & -11.7 & -11.7 & -8.5 \\
\hline 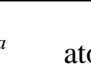 & & & & & & & \\
\hline $\mathrm{Lc}$ & $(\mathrm{eV}$ & e sp & ridized c & tal need & $f$ & tio & rganic \\
\hline
\end{tabular}

(S2) Becke, A. D. Phys. Rev. A: At., Mol., Opt. Phys. 1988, 38, 3098-3100.

(S3) Perdew, J. P. Phys. Rev. B: Condens. Matter Mater. Phys. 1986, 33, 8822-8824.

(S4) Frisch, M. J.; Pople, J. A.; Binkley, J. S. J. Chem. Phys. 1984, 80, 3265-3269. 


\section{Absolute Energies of Calculated Structures}

All energies were calculated at the BP86/6-311++G(3df,3pd) level of theory corrected for zero point energy $(\Delta U)$ as well as thermodynamic effects $(\Delta G)$ using unscaled frequencies and standard temperature $(298.15 \mathrm{~K})$ and pressure $(1 \mathrm{~atm})$. Solvent effects were incorporated by the C-PCM model using acetonitrile $(\varepsilon=36.64)$ as solvent. $N_{\text {imag }}$ denotes the number of imaginary frequencies.

Table S4: Absolute energies in solution [a.u.]

\begin{tabular}{|c|c|c|c|c|}
\hline Compound & $E_{S C F}$ & $\Delta U$ & $\Delta G$ & $N_{i m a g}$ \\
\hline 10 & -686.962476013 & -686.836848 & -686.873034 & 0 \\
\hline 11 & -571.571266547 & -571.489161 & -571.519480 & 0 \\
\hline 12 & -381.882991844 & -381.781168 & -381.813472 & 0 \\
\hline $14 \mathbf{a}$ & -909.835944419 & -909.685533 & -909.725192 & 0 \\
\hline 14b & -909.829091547 & -909.688961 & -909.728939 & 0 \\
\hline 15 & -910.255750309 & -910.104077 & -910.144967 & 0 \\
\hline 16 & -1139.03397000 & -1138.833047 & -1138.879485 & 0 \\
\hline 17 & -1025.62987298 & -1025.424011 & -1025.470827 & 0 \\
\hline 18 & -1025.60576013 & -1025.414868 & -1025.459526 & 0 \\
\hline 19 & -1025.61214736 & -1025.406188 & -1025.449945 & 0 \\
\hline 20 & -802.253784082 & -802.088001 & -802.128819 & 0 \\
\hline 21 & -686.953307579 & -686.834158 & -686.870009 & 0 \\
\hline 22 & -800.349384426 & -800.220068 & -800.257708 & 0 \\
\hline 23 & -800.341946316 & -800.212207 & -800.247940 & 0 \\
\hline 2d & -566.615659920 & -566.497371 & -566.536266 & 0 \\
\hline 3d & -909.813942497 & -909.665735 & -909.706077 & 0 \\
\hline 4 & -910.272667305 & -910.111625 & -910.151918 & 0 \\
\hline 5 & -573.551796686 & -573.437388 & -573.469160 & 0 \\
\hline 6 & -567.883269122 & -567.740973 & -567.779551 & 0 \\
\hline 7 & -458.172367348 & -458.100238 & -458.127741 & 0 \\
\hline 8 & -720.580600827 & -720.398543 & -720.441693 & 0 \\
\hline 9 & -268.494905633 & -268.403711 & -268.433832 & 0 \\
\hline $\mathrm{AcOH}$ & -229.187424534 & -229.124436 & -229.151828 & 0 \\
\hline $\mathrm{AcO}^{-}$ & -228.722072897 & -228.672413 & -228.700242 & 0 \\
\hline $\mathbf{E}_{15-7}$ & -1026.04261684 & -1025.837744 & -1025.881443 & 0 \\
\hline $\mathbf{E}_{3 d-17}$ & -1025.60601413 & -1025.416132 & -1025.463744 & 0 \\
\hline $\mathbf{H}_{3} \mathbf{P}=\mathbf{O}$ & -418.468087544 & -418.434720 & -418.458356 & 0 \\
\hline МeOH & -115.774366174 & -115.722937 & -115.745705 & 0 \\
\hline $\mathrm{MeO}^{-}$ & -115.273983165 & -115.237632 & -115.258883 & 0 \\
\hline $\mathbf{P H}_{3}$ & -343.194058188 & -343.167121 & -343.188180 & 0 \\
\hline
\end{tabular}


Table S4: (continued)

\begin{tabular}{ccrrc}
\hline Compound & $E_{S C F}$ & $\Delta U$ & $\Delta G$ & $N_{\text {imag }}$ \\
\hline $\mathbf{T}_{\mathbf{1 5 - 7}}$ & -1026.01190589 & -1025.810167 & -1025.853588 & 1 \\
$\mathbf{T}_{\mathbf{1 6 - 8}}$ & -1139.01337092 & -1138.813647 & -1138.859968 & 1 \\
$\mathbf{T}_{\mathbf{1 7 - 5}}$ & -1141.40157750 & -1141.137570 & -1141.182015 & 1 \\
$\mathbf{T}_{\mathbf{2 0 - 1 0}}$ & -802.248438848 & -802.091586 & -802.131022 & 1 \\
$\mathbf{T}_{\mathbf{2 1 - 9 R e t}}$ & -686.918691118 & -686.800884 & -686.834122 & 1 \\
$\mathbf{T}_{\mathbf{2 2 - 2 3}}$ & -800.340154516 & -800.210447 & -800.245095 & 1 \\
$\mathbf{T}_{\mathbf{2 3 - 1 2}}$ & -800.332785801 & -800.206220 & -800.242639 & 1 \\
$\mathbf{T}_{\mathbf{3 d - 1 7}}$ & -1025.60368840 & -1025.415206 & -1025.459890 & 1 \\
$\mathbf{T}_{\mathbf{4 - 7}}$ & -1026.01679063 & -1025.804197 & -1025.848574 & 1 \\
$\mathbf{T}_{\text {5-20 }}$ & -802.260327981 & -802.102993 & -802.148256 & 1 \\
$\mathbf{T}_{\text {7-9Inv }}$ & -686.895676091 & -686.772652 & -686.811787 & 1 \\
$\mathbf{T}_{\text {7-9Ret }}$ & -686.872544124 & -686.758036 & -686.794966 & 1 \\
\hline
\end{tabular}

Table S5: Absolute energies in the gas phase [a. u.]

\begin{tabular}{ccccc}
\hline Compound & $E_{S C F}$ & $\Delta U$ & $\Delta G$ & $N_{\text {imag }}$ \\
\hline $\mathbf{1 0}$ & -686.958687524 & -686.839277 & -686.875009 & 0 \\
$\mathbf{1 1}$ & -571.502014104 & -571.424694 & -571.455174 & 0 \\
$\mathbf{1 2}$ & -381.876541734 & -381.781800 & -381.815428 & 0 \\
$\mathbf{1 3}$ & -909.796469553 & -909.657466 & -909.698616 & 0 \\
$\mathbf{1 4 a}$ & -909.831315114 & -909.690093 & -909.730247 & 0 \\
$\mathbf{1 4 b}$ & -909.831315114 & -909.690093 & -909.730247 & 0 \\
$\mathbf{1 5}$ & -910.171945786 & -910.018370 & -910.058931 & 0 \\
$\mathbf{1 6}$ & -1139.00497822 & -1138.802758 & -1138.851994 & 0 \\
$\mathbf{1 7}$ & -1025.62124551 & -1025.427602 & -1025.476206 & 0 \\
$\mathbf{1 8}$ & -1025.58567132 & -1025.392967 & -1025.438558 & 0 \\
$\mathbf{1 9}$ & -1025.60451858 & -1025.409010 & -1025.453171 & 0 \\
$\mathbf{2 0}$ & -802.183945930 & -802.024880 & -802.065078 & 0 \\
$\mathbf{2 1}$ & -686.944451925 & -686.824800 & -686.860448 & 0 \\
$\mathbf{2 2}$ & -800.342188385 & -800.212097 & -800.249620 & 0 \\
$\mathbf{2 3}$ & -800.334179030 & -800.204471 & -800.239991 & 0 \\
$\mathbf{2 d}$ & -566.609079126 & -566.499535 & -566.538826 & 0 \\
$\mathbf{3 d}$ & -909.795762620 & -909.657323 & -909.698223 & 0 \\
$\mathbf{4}$ & -910.208202376 & -910.055160 & -910.095611 & 0 \\
$\mathbf{5}$ & -573.548901011 & -573.439130 & -573.470943 & 0 \\
$\mathbf{6}$ & -567.872363778 & -567.737693 & -567.776277 & 0 \\
\hline
\end{tabular}


Table S5: (continued)

\begin{tabular}{|c|c|c|c|c|}
\hline Compound & $E_{S C F}$ & $\Delta U$ & $\Delta G$ & $N_{\text {imag }}$ \\
\hline 7 & -458.100341864 & -458.031684 & -458.059247 & 0 \\
\hline 8 & -720.569686790 & -720.399619 & -720.444087 & 0 \\
\hline 9 & -268.491336229 & -268.404775 & -268.435505 & 0 \\
\hline $\mathrm{AcO}^{-}$ & -228.620357372 & -228.574103 & -228.601643 & 0 \\
\hline $\mathrm{AcOH}$ & -229.181758241 & -229.122084 & -229.149361 & 0 \\
\hline $\mathbf{E}_{15-7}$ & -1025.95914704 & -1025.752427 & -1025.798788 & 0 \\
\hline $\mathrm{H}_{3} \mathrm{P}=\mathrm{O}$ & -418.463508268 & -418.433817 & -418.457448 & 0 \\
\hline $\mathrm{MeO}^{-}$ & -115.157069645 & -115.123885 & -115.145103 & 0 \\
\hline МеOH & -115.770324916 & -115.720726 & -115.743543 & 0 \\
\hline $\mathbf{P H}_{3}$ & -343.193832543 & -343.170667 & -343.191727 & 0 \\
\hline $\mathbf{T}_{15-7}$ & -1025.93554723 & -1025.731700 & -1025.775161 & 1 \\
\hline $\mathbf{T}_{16-8}$ & -1138.98892211 & -1138.788281 & -1138.836102 & 1 \\
\hline $\mathbf{T}_{17-5}$ & -1141.39204993 & -1141.145753 & -1141.196938 & 1 \\
\hline$T_{21-9 R e t}$ & -686.904587452 & -686.786691 & -686.820323 & 1 \\
\hline $\mathbf{T}_{22-23}$ & -800.333810816 & -800.203349 & -800.237991 & 1 \\
\hline $\mathbf{T}_{23-12}$ & -800.321256574 & -800.193812 & -800.229888 & 1 \\
\hline $\mathbf{T}_{4-7}$ & -1025.95492856 & -1025.752536 & -1025.796909 & 1 \\
\hline $\mathbf{T}_{5-20}$ & -802.166359209 & -802.009434 & -802.051887 & 1 \\
\hline$T_{\text {7-9Inv }}$ & -686.881234183 & -686.764947 & -686.800884 & 1 \\
\hline $\mathbf{T}_{\text {7-9Ret }}$ & -686.860442124 & -686.745277 & -686.781510 & 1 \\
\hline
\end{tabular}




\section{Cartesian Coordinates of Calculated Structures}

All cartesian coordinates are given in Ångström. For convenience, coordinates of all compounds are also available in xyz-format packed together into a single ZIP-archive.

\subsection{Coordinates in Solution}

All structures were fully optimized at the BP86/6-311++G(3df,3pd) level of theory using the C-PCM solvation model and acetonitrile $(\varepsilon=36.64)$ as solvent.

10

16

P $\quad-0.759277 \quad-0.189987 \quad-0.221506$

$\begin{array}{llll}\text { O } & 1.598025 & 1.355692 & 0.088213\end{array}$

O $\quad 0.966023 \quad-0.796369-0.064175$

O $\quad-2.342751 \quad 0.341993 \quad-0.468172$

$\begin{array}{llll}\text { C } & 3.297297 & -0.371696 & 0.165272\end{array}$

$\begin{array}{llll}\text { C } & 1.889419 & 0.159972 & 0.061606\end{array}$

C $\quad-3.284263 \quad-0.056061 \quad 0.525766$

$\mathrm{H} \quad 4.007663 \quad 0.453922 \quad 0.270356$

$\mathrm{H} \quad 3.542245 \quad-0.960965-0.729315$

$\begin{array}{llll}\mathrm{H} & 3.381043 & -1.046644 & 1.028338\end{array}$

$\mathrm{H} \quad-4.258359 \quad 0.373855 \quad 0.262773$

$\mathrm{H} \quad-3.383302 \quad-1.156043 \quad 0.571125$

$\begin{array}{llll}\mathrm{H} & -2.993776 & 0.313612 & 1.526534\end{array}$

$\mathrm{H} \quad-1.060243 \quad-1.569163 \quad-0.247747$

$\begin{array}{llll}\mathrm{H} & -0.645464 & 0.359057 & 1.074807\end{array}$

$\begin{array}{llll}\mathrm{H} & -0.385735 & 0.478355 & -1.397068\end{array}$

\section{1}

11

$\begin{array}{llll}\mathrm{P} & 1.581378 & -0.076035 & 0.000841\end{array}$

$\begin{array}{llll}\text { O } & 0.148450 & -0.804946 & -0.002615\end{array}$

$\begin{array}{llll}\mathrm{O} & -0.549207 & 1.334084 & -0.000641\end{array}$

$\begin{array}{llll}\text { C } & -2.244907 & -0.436924 & 0.001196\end{array}$

$\begin{array}{llll}\text { C } & -0.905396 & 0.183003 & -0.001292\end{array}$

$\begin{array}{llll}\mathrm{H} & 2.473066 & -1.163629 & 0.002816\end{array}$

$\begin{array}{llll}\mathrm{H} & 1.813697 & 0.707198 & -1.146760\end{array}$ 


$\begin{array}{rrrr}\mathrm{H} & 1.808426 & 0.706928 & 1.149718 \\ \mathrm{H} & -3.009681 & 0.344649 & -0.004826 \\ \mathrm{H} & -2.349528 & -1.074867 & 0.891538 \\ \mathrm{H} & -2.348766 & -1.089332 & -0.878476\end{array}$

\section{2}

13

$\begin{array}{llll}\mathrm{O} & 1.293595 & 1.200419 & -0.601234\end{array}$

$\begin{array}{llll}\mathrm{O} & -1.293525 & 1.200446 & 0.601239\end{array}$

$\begin{array}{llll}\text { O } & -0.000018 & -0.596934 & -0.000071\end{array}$

$\begin{array}{llll}\text { C } & 2.346858 & -0.786753 & 0.304465\end{array}$

$\begin{array}{llll}\text { C } & 1.216096 & 0.090901 & -0.140355\end{array}$

$\begin{array}{llll}\text { C } & -2.346905 & -0.786708 & -0.304442\end{array}$

$\begin{array}{llll}\text { C } & -1.216091 & 0.090937 & 0.140336\end{array}$

$\begin{array}{llll}\mathrm{H} & 3.302848 & -0.300108 & 0.091071\end{array}$

H $\quad 2.294318 \quad-1.759759 \quad-0.202614$

H $\quad 2.258001 \quad-0.980963 \quad 1.383270$

$\mathrm{H} \quad-3.302837 \quad-0.299348 \quad-0.092426$

$\begin{array}{llll}\mathrm{H} & -2.295271 & -1.759023 & 0.204084\end{array}$

H $\quad-2.257219 \quad-0.982505 \quad-1.382875$

\section{$14 a$}

20

$\begin{array}{lrrr}\mathrm{P} & -0.187294 & 1.804836 & 0.110483 \\ \mathrm{O} & 3.110259 & -0.229790 & -0.102627 \\ \mathrm{O} & 1.617151 & 1.434206 & -0.178242 \\ \mathrm{O} & -2.618893 & 0.101778 & -0.133778 \\ \mathrm{O} & -1.564325 & -1.894111 & 0.153572 \\ \mathrm{~N} & 0.844365 & -0.697464 & 0.085159 \\ \mathrm{~N} & -0.345883 & 0.071719 & 0.071515 \\ \mathrm{C} & 3.357989 & -1.650602 & 0.000326 \\ \mathrm{C} & 1.828151 & 0.140867 & -0.060715 \\ \mathrm{C} & -3.875964 & -0.614538 & -0.137952 \\ \mathrm{C} & -1.530610 & -0.684590 & 0.046320 \\ \mathrm{H} & 4.444700 & -1.757987 & -0.062302 \\ \mathrm{H} & 2.986990 & -2.035044 & 0.959177 \\ \mathrm{H} & 2.868952 & -2.185189 & -0.824175\end{array}$




$\begin{array}{rrrr}\mathrm{H} & 0.205785 & 2.485504 & 1.290200 \\ \mathrm{H} & -4.640975 & 0.154885 & -0.277427 \\ \mathrm{H} & -4.024198 & -1.136945 & 0.814940 \\ \mathrm{H} & -3.901767 & -1.337689 & -0.962035 \\ \mathrm{H} & -1.584287 & 1.993327 & 0.303701 \\ \mathrm{H} & -0.066095 & 2.683321 & -0.995310\end{array}$

$14 b$

20

$\begin{array}{lrrr}\mathrm{P} & -0.045105 & 1.607306 & -0.282716 \\ \mathrm{O} & 2.597896 & 0.376461 & 0.119476 \\ \mathrm{O} & 1.905848 & -1.784114 & -0.145362 \\ \mathrm{O} & -2.776573 & -1.099558 & 0.080457 \\ \mathrm{O} & -1.474767 & 0.666807 & 0.757789 \\ \mathrm{~N} & 0.467268 & -0.021356 & -0.547148 \\ \mathrm{~N} & -0.639466 & -0.915983 & -0.674058 \\ \mathrm{C} & 3.881682 & -0.136690 & 0.574933 \\ \mathrm{C} & 1.692085 & -0.585384 & -0.177996 \\ \mathrm{C} & -3.941327 & -0.252178 & 0.274268 \\ \mathrm{C} & -1.604595 & -0.436832 & 0.083264 \\ \mathrm{H} & 4.503039 & 0.746189 & 0.748480 \\ \mathrm{H} & 4.330039 & -0.783863 & -0.188641 \\ \mathrm{H} & 3.749287 & -0.703459 & 1.505742 \\ \mathrm{H} & 0.907451 & 2.298963 & -1.090382 \\ \mathrm{H} & 0.089026 & 2.350765 & 0.912182 \\ \mathrm{H} & -4.803992 & -0.912844 & 0.153374 \\ \mathrm{H} & -3.961254 & 0.536042 & -0.492287 \\ \mathrm{H} & -3.936494 & 0.202423 & 1.271748 \\ \mathrm{H} & -1.181452 & 1.907293 & -1.056745\end{array}$

15

21

$\begin{array}{llll}\mathrm{P} & 0.188206 & 2.171819 & -0.307340\end{array}$

$\begin{array}{llll}\text { O } & 2.632540 & -0.656825 & -0.360147\end{array}$

$\begin{array}{llll}\mathrm{O} & 1.360656 & 1.100810 & -0.664643\end{array}$

$\begin{array}{llll}\text { O } & -2.767294 & 0.301703 & 0.857722\end{array}$ 


$\begin{array}{rrrr}\mathrm{O} & -1.565840 & -1.151073 & -0.434207 \\ \mathrm{~N} & 0.659737 & -0.586890 & 0.840950 \\ \mathrm{~N} & -0.459870 & 0.302803 & 0.941383 \\ \mathrm{C} & 2.933017 & -1.955525 & 0.240187 \\ \mathrm{C} & 1.503763 & -0.099952 & 0.001044 \\ \mathrm{C} & -2.797489 & -1.690521 & -1.000726 \\ \mathrm{C} & -1.728719 & -0.200019 & 0.478190 \\ \mathrm{H} & 3.884898 & -2.250656 & -0.208127 \\ \mathrm{H} & 3.023475 & -1.843922 & 1.327001 \\ \mathrm{H} & 2.137976 & -2.667985 & -0.008170 \\ \mathrm{H} & 0.731200 & 3.186163 & -1.133574 \\ \mathrm{H} & 0.177658 & 2.767379 & 0.973117 \\ \mathrm{H} & -3.397936 & -2.148110 & -0.205851 \\ \mathrm{H} & -3.363385 & -0.890644 & -1.492465 \\ \mathrm{H} & -2.468399 & -2.440829 & -1.724192 \\ \mathrm{H} & -1.112080 & 2.010731 & -0.834434 \\ \mathrm{H} & -0.579502 & 0.608373 & 1.938498\end{array}$

16

28

$\begin{array}{llll}\mathrm{P} & 0.826915 & -1.467400 & -0.748881\end{array}$

$\begin{array}{llll}\text { O } & 4.312541 & 0.411515 & -0.195746\end{array}$

$\begin{array}{llll}\mathrm{O} & 2.351143 & -0.416194 & -0.818544\end{array}$

$\begin{array}{llll}\mathrm{O} & 0.438637 & 1.535713 & -1.284189\end{array}$

$\begin{array}{llll}\text { O } & -3.224540 & -1.038629 & 1.475243\end{array}$

$\begin{array}{llll}\text { O } & -2.537033 & -0.466614 & -0.633329\end{array}$

$\begin{array}{llll}\mathrm{O} & -0.702554 & 1.874554 & 0.661643\end{array}$

$\begin{array}{llll}\mathrm{N} & -1.010329 & -0.699056 & 1.016561\end{array}$

$\begin{array}{llll}\mathrm{N} & -0.022127 & -0.226796 & 0.158648\end{array}$

$\begin{array}{llll}\text { C } & 3.283722 & -0.187646 & 0.112442\end{array}$

$\begin{array}{llll}\text { C } & 3.035948 & -0.684806 & 1.520688\end{array}$

$\begin{array}{llll}\text { C } & -3.912435 & -0.537178 & -1.087001\end{array}$

$\begin{array}{llll}\text { C } & -2.349057 & -0.749170 & 0.671650\end{array}$

$\begin{array}{llll}\text { C } & -0.864598 & 3.272037 & 0.300097\end{array}$

$\begin{array}{llll}\text { C } & -0.053008 & 1.128629 & -0.249330\end{array}$

$\begin{array}{llll}\mathrm{H} & 3.873715 & -0.399860 & 2.164426\end{array}$

H $\quad 2.936092 \quad-1.781195 \quad 1.533633$

$\begin{array}{lllr}\mathrm{H} & 2.105031 & -0.264999 & 1.926944\end{array}$

H $\quad 1.599449 \quad-2.437111 \quad-0.063814$ 


$\begin{array}{rrrr}\mathrm{H} & 0.967424 & -1.455351 & -2.154258 \\ \mathrm{H} & 0.115638 & 3.758858 & 0.228955 \\ \mathrm{H} & -4.530265 & 0.186251 & -0.540987 \\ \mathrm{H} & -4.308215 & -1.549947 & -0.942391 \\ \mathrm{H} & -3.874695 & -0.286431 & -2.151059 \\ \mathrm{H} & -1.455460 & 3.707796 & 1.110787 \\ \mathrm{H} & -1.394357 & 3.356646 & -0.656551 \\ \mathrm{H} & -0.792105 & -0.732343 & 2.026788 \\ \mathrm{H} & -0.367762 & -2.264289 & -0.727617\end{array}$

17

26

$\begin{array}{lrrr}\mathrm{P} & -1.521358 & -1.107247 & 0.539000 \\ \mathrm{O} & 2.782335 & -1.294911 & -1.387380 \\ \mathrm{O} & 1.979819 & -0.880769 & 0.717043 \\ \mathrm{O} & 0.755888 & 2.084229 & -0.426704 \\ \mathrm{O} & -2.656195 & -0.473294 & -0.462092 \\ \mathrm{O} & -1.093964 & 1.861896 & 0.904301 \\ \mathrm{~N} & 0.677081 & -0.454533 & -1.080715 \\ \mathrm{~N} & -0.309074 & 0.069488 & -0.264574 \\ \mathrm{C} & 3.233346 & -1.368291 & 1.242151 \\ \mathrm{C} & 1.898073 & -0.904343 & -0.636640 \\ \mathrm{C} & 0.812393 & 3.469155 & -0.025034 \\ \mathrm{C} & -4.029925 & -0.905213 & -0.336128 \\ \mathrm{C} & -0.272035 & 1.384100 & 0.127390 \\ \mathrm{H} & 4.068568 & -0.756957 & 0.878242 \\ \mathrm{H} & 3.393778 & -2.414112 & 0.951463 \\ \mathrm{H} & 3.143893 & -1.283687 & 2.329354 \\ \mathrm{H} & 1.669097 & 3.891103 & -0.559788 \\ \mathrm{H} & 0.959988 & 3.553967 & 1.059103 \\ \mathrm{H} & 0.565234 & -0.371994 & -2.091704 \\ \mathrm{H} & -4.567106 & -0.420299 & -1.159253 \\ \mathrm{H} & -4.461255 & -0.582371 & 0.621661 \\ \mathrm{H} & -4.122539 & -1.995872 & -0.431770 \\ \mathrm{H} & -2.481901 & -2.053301 & 1.084751 \\ \mathrm{H} & -1.202090 & -0.556315 & 1.805324 \\ \mathrm{H} & -0.605771 & -2.128660 & 0.160278 \\ \mathrm{H} & -0.109748 & 3.992853 & -0.307420\end{array}$


18

26

$\begin{array}{llll}\mathrm{P} & 0.601544 & -1.999671 & 0.081022\end{array}$

$\begin{array}{llll}\text { O } & 1.868839 & -1.116209 & -0.452727\end{array}$

$\begin{array}{llll}\mathrm{O} & 1.277300 & 2.629428 & 0.390761\end{array}$

$\begin{array}{llll}\text { O } & 0.841084 & 0.866275 & 1.695263\end{array}$

$\begin{array}{llll}\mathrm{O} & -2.497508 & 0.559239 & -0.239953\end{array}$

$\begin{array}{llll}\mathrm{O} & -2.295134 & -1.696190 & 0.116438\end{array}$

$\begin{array}{llll}\mathrm{N} & 0.061820 & 0.887383 & -0.506392\end{array}$

$\mathrm{N} \quad-0.428636 \quad-0.416450 \quad-0.224214$

$\begin{array}{llll}\text { C } & 3.194950 & -1.687866 & -0.436230\end{array}$

$\begin{array}{llll}\text { C } & 1.161359 & 3.314830 & -0.878294\end{array}$

$\begin{array}{llll}\text { C } & 0.688437 & 1.423367 & 0.484315\end{array}$

$\begin{array}{llll}\text { C } & -3.923350 & 0.383508 & -0.109587\end{array}$

$\begin{array}{llll}\text { C } & -1.767270 & -0.591474 & -0.104322\end{array}$

$\begin{array}{llll}\mathrm{H} & 3.846682 & -0.924011 & -0.876161\end{array}$

$\begin{array}{llll}\mathrm{H} & 3.522430 & -1.903703 & 0.590222\end{array}$

$\begin{array}{llll}\mathrm{H} & 3.244647 & -2.604684 & -1.039816\end{array}$

H $\quad 1.706521 \quad 4.254645 \quad-0.745935$

$\begin{array}{llll}\mathrm{H} & 1.610487 & 2.711762 & -1.677460\end{array}$

$\begin{array}{llll}\mathrm{H} & 1.458178 & -3.154539 & 0.308562\end{array}$

$\begin{array}{lllr}\mathrm{H} & 1.386270 & 1.467078 & 2.277847\end{array}$

H $\quad 0.106475 \quad 3.506147 \quad-1.111840$

$\begin{array}{llll}\mathrm{H} & 0.081174 & -2.036681 & 1.399110\end{array}$

$\begin{array}{llll}\mathrm{H} & -4.349419 & 1.384026 & -0.239765\end{array}$

$\begin{array}{llll}\mathrm{H} & -4.309571 & -0.296104 & -0.881025\end{array}$

$\begin{array}{llll}\mathrm{H} & -4.181023 & -0.012371 & 0.881813\end{array}$

$\begin{array}{llll}\mathrm{H} & -0.259717 & -2.687573 & -0.800184\end{array}$

19

26

$\begin{array}{lllr}\mathrm{P} & -0.136271 & 1.371649 & 0.964258\end{array}$

$\begin{array}{llll}\text { O } & 2.769016 & -0.175365 & -0.688768\end{array}$

$\begin{array}{llll}\text { O } & 1.925212 & -1.640460 & 0.851407\end{array}$

$\begin{array}{llll}\text { O } & -2.774916 & -1.331045 & 0.103349\end{array}$

$\begin{array}{llll}\mathrm{O} & -1.731891 & 0.579705 & 0.431964\end{array}$

$\begin{array}{llll}\text { O } & -0.150669 & 2.345130 & -0.402960\end{array}$ 


$\begin{array}{lrrr}\mathrm{N} & 0.535964 & -0.318686 & -0.473270 \\ \mathrm{~N} & -0.540195 & -1.258492 & -0.416680 \\ \mathrm{C} & 4.109003 & -0.498554 & -0.244863 \\ \mathrm{C} & 1.784620 & -0.820587 & -0.028823 \\ \mathrm{C} & -2.767835 & -2.698142 & -0.366650 \\ \mathrm{C} & -1.617610 & -0.659863 & 0.029359 \\ \mathrm{C} & -1.368087 & 2.913663 & -0.899629 \\ \mathrm{H} & 4.772595 & 0.112001 & -0.863507 \\ \mathrm{H} & 4.311298 & -1.565499 & -0.398297 \\ \mathrm{H} & 4.230056 & -0.247651 & 0.815897 \\ \mathrm{H} & 1.228415 & 1.751780 & 1.141406 \\ \mathrm{H} & 0.611841 & 0.134475 & -1.392750 \\ \mathrm{H} & -3.799466 & -3.043887 & -0.249592 \\ \mathrm{H} & -2.462548 & -2.741405 & -1.419724 \\ \mathrm{H} & -2.085912 & -3.309646 & 0.237277 \\ \mathrm{H} & -2.012924 & 3.265960 & -0.078090 \\ \mathrm{H} & -1.932139 & 2.196318 & -1.512239 \\ \mathrm{H} & -1.079460 & 3.773621 & -1.518437 \\ \mathrm{H} & -0.803344 & 2.249002 & 1.857235 \\ \mathrm{H} & -0.039301 & 0.247622 & 1.850306\end{array}$

20

21

$\begin{array}{lrrr}\mathrm{P} & -0.506553 & -0.463700 & -0.122951 \\ \mathrm{O} & 3.494113 & 0.316261 & -0.712666 \\ \mathrm{O} & 1.274066 & 0.113102 & -0.760972 \\ \mathrm{O} & -2.022977 & -1.100735 & 0.400480 \\ \mathrm{O} & -1.088094 & 1.178577 & -0.430474 \\ \mathrm{C} & 2.470659 & -0.703908 & 1.196720 \\ \mathrm{C} & 2.429646 & -0.054780 & -0.181175 \\ \mathrm{C} & -3.180820 & -0.747178 & -0.328522 \\ \mathrm{C} & -1.325546 & 2.023122 & 0.670072 \\ \mathrm{H} & 3.484231 & -1.065304 & 1.403587 \\ \mathrm{H} & 2.206334 & 0.042199 & 1.960377 \\ \mathrm{H} & 1.749570 & -1.528488 & 1.279872 \\ \mathrm{H} & 0.101168 & -1.773098 & 0.048699 \\ \mathrm{H} & -3.994125 & -1.425270 & -0.024572 \\ \mathrm{H} & -3.500307 & 0.293751 & -0.137460 \\ \mathrm{H} & -3.033421 & -0.856270 & -1.419978\end{array}$




$\begin{array}{lrrr}\mathrm{H} & -2.391212 & 2.019706 & 0.974386 \\ \mathrm{H} & -1.046032 & 3.058519 & 0.410686 \\ \mathrm{H} & -0.745526 & -0.652835 & -1.523615 \\ \mathrm{H} & -0.730391 & 1.725779 & 1.558177 \\ \mathrm{H} & -0.122496 & -0.044360 & 1.200589\end{array}$

21

16

$\begin{array}{llll}\mathrm{P} & -0.919115 & 0.925912 & 0.255804\end{array}$

$\begin{array}{llll}\text { O } & 1.293536 & -0.870713 & 1.104541\end{array}$

$\begin{array}{llll}\text { O } & 0.735265 & 0.623304 & -0.498049\end{array}$

$\begin{array}{llll}\mathrm{O} & -1.350183 & -0.490250 & -0.438518\end{array}$

$\begin{array}{llll}\text { C } & 2.954507 & -0.217446 & -0.533283\end{array}$

$\begin{array}{llll}\text { C } & 1.585869 & -0.203134 & 0.112001\end{array}$

$\begin{array}{llll}\text { C } & -2.706164 & -0.974648 & -0.271132\end{array}$

H $\quad 3.601594 \quad-0.946258 \quad-0.035119$

$\begin{array}{llll}\mathrm{H} & 3.405449 & 0.783118 & -0.467117\end{array}$

$\begin{array}{llll}\mathrm{H} & 2.865642 & -0.462999 & -1.600590\end{array}$

$\begin{array}{llll}\mathrm{H} & -3.437986 & -0.255285 & -0.663235\end{array}$

$\mathrm{H} \quad-2.921348 \quad-1.187845 \quad 0.784791$

$\begin{array}{llll}\mathrm{H} & -2.756029 & -1.902885 & -0.851657\end{array}$

$\begin{array}{llll}\mathrm{H} & -2.248600 & 1.147776 & 0.779453\end{array}$

$\mathrm{H} \quad-0.874759 \quad 2.130805 \quad-0.481974$

$\mathrm{H} \quad-0.281456 \quad 1.077529 \quad 1.509094$

22

18

$\begin{array}{llll}\mathrm{P} & 0.413461 & 1.597193 & -0.000031\end{array}$

$\begin{array}{llll}\mathrm{O} & 1.142781 & 0.167414 & -1.072290\end{array}$

$\begin{array}{lllr}\text { O } & 1.142742 & 0.166923 & 1.072760\end{array}$

$\begin{array}{llll}\mathrm{O} & -1.191215 & 0.763471 & -0.000267\end{array}$

$\begin{array}{llll}\text { O } & -0.898739 & -1.503341 & -0.000503\end{array}$

$\begin{array}{llll}\text { C } & 2.240477 & -1.731977 & -0.000110\end{array}$

$\begin{array}{llll}\text { C } & 1.466225 & -0.466746 & 0.000146\end{array}$

$\begin{array}{llll}\text { C } & -3.128535 & -0.574715 & 0.000303\end{array}$

$\begin{array}{llll}\text { C } & -1.615865 & -0.507469 & -0.000345\end{array}$

$\mathrm{H} \quad 3.313514 \quad-1.481669 \quad 0.003291$ 


$\begin{array}{lrrr}\mathrm{H} & 2.020675 & -2.311070 & -0.902837 \\ \mathrm{H} & 2.015899 & -2.314145 & 0.899486 \\ \mathrm{H} & 1.735611 & 2.131141 & 0.000342 \\ \mathrm{H} & -3.526586 & -0.053424 & -0.881365 \\ \mathrm{H} & -3.524660 & -0.062163 & 0.888022 \\ \mathrm{H} & -3.457925 & -1.618589 & -0.004139 \\ \mathrm{H} & -0.058562 & 2.340651 & 1.105957 \\ \mathrm{H} & -0.058247 & 2.341073 & -1.105858\end{array}$

23

18

$\begin{array}{llll}\mathrm{P} & 1.320990 & -1.280274 & 0.002208\end{array}$

$\begin{array}{llll}\mathrm{O} & 0.998745 & 0.084309 & -1.068359\end{array}$

$\begin{array}{llll}\mathrm{O} & 0.991040 & 0.083645 & 1.070281\end{array}$

$\begin{array}{llll}\mathrm{O} & -1.049026 & -1.293658 & -0.006291\end{array}$

$\begin{array}{llll}\text { O } & -0.934703 & 0.975174 & -0.005786\end{array}$

$\begin{array}{llll}\text { C } & 1.081286 & 2.303372 & 0.001615\end{array}$

$\begin{array}{llll}\text { C } & 0.606093 & 0.874961 & -0.000300\end{array}$

$\begin{array}{llll}\text { C } & -3.089019 & -0.004332 & 0.003663\end{array}$

$\begin{array}{llll}\text { C } & -1.596291 & -0.181741 & -0.005577\end{array}$

$\begin{array}{llll}\mathrm{H} & 2.733475 & -1.268079 & 0.006678\end{array}$

$\begin{array}{llll}\mathrm{H} & 2.178109 & 2.306751 & 0.005210\end{array}$

H $\quad 1.079599 \quad-2.193575 \quad-1.058486$

$\begin{array}{llll}\mathrm{H} & 1.072832 & -2.194029 & 1.060985\end{array}$

$\begin{array}{llll}\mathrm{H} & 0.718448 & 2.822672 & -0.893409\end{array}$

$\begin{array}{llrr}\mathrm{H} & 0.712718 & 2.822165 & 0.894591\end{array}$

$\begin{array}{llll}\mathrm{H} & -3.582907 & -0.977251 & -0.079259\end{array}$

$\mathrm{H} \quad-3.395542 \quad 0.649361 \quad-0.824231$

$\begin{array}{llll}\mathrm{H} & -3.392424 & 0.486776 & 0.939633\end{array}$

2d

16

$\begin{array}{llrr}\text { O } & 2.423852 & 0.683440 & 0.117318\end{array}$

$\begin{array}{llll}\text { O } & 2.140310 & -1.577830 & -0.069542\end{array}$

O $\quad-2.422733 \quad-0.683855 \quad-0.113531$

$\begin{array}{llll}\mathrm{O} & -2.142138 & 1.578556 & 0.063647\end{array}$ 


$\begin{array}{rrrr}\mathrm{N} & 0.349449 & -0.218615 & 0.467452 \\ \mathrm{~N} & -0.349748 & 0.219796 & -0.469375 \\ \mathrm{C} & 3.860150 & 0.535964 & -0.080456 \\ \mathrm{C} & 1.732753 & -0.451125 & 0.102594 \\ \mathrm{C} & -3.859012 & -0.537479 & 0.084934 \\ \mathrm{C} & -1.733162 & 0.451737 & -0.104370 \\ \mathrm{H} & 4.270920 & -0.150531 & 0.669025 \\ \mathrm{H} & 4.269320 & 1.541700 & 0.044031 \\ \mathrm{H} & 4.058540 & 0.155994 & -1.089237 \\ \mathrm{H} & -4.271408 & 0.145298 & -0.667049 \\ \mathrm{H} & -4.266956 & -1.544275 & -0.034942 \\ \mathrm{H} & -4.057011 & -0.153521 & 1.092282\end{array}$

3d

20

$\begin{array}{lrrr}\mathrm{P} & -0.026311 & 1.875941 & -0.360152 \\ \mathrm{O} & 2.137441 & 1.042310 & 0.497193 \\ \mathrm{O} & 2.053021 & -1.190516 & -0.045135 \\ \mathrm{O} & -2.527016 & -1.677139 & -0.681120 \\ \mathrm{O} & -1.765596 & 0.046829 & 0.633313 \\ \mathrm{~N} & 0.363414 & 0.252558 & -0.609622 \\ \mathrm{~N} & -0.500279 & -0.713128 & -1.162995 \\ \mathrm{C} & 3.354120 & -1.377443 & 0.579405 \\ \mathrm{C} & 1.588499 & 0.046290 & -0.003270 \\ \mathrm{C} & -3.015184 & -0.034777 & 1.341196 \\ \mathrm{C} & -1.634215 & -0.864500 & -0.439393 \\ \mathrm{H} & 4.108109 & -0.770468 & 0.065209 \\ \mathrm{H} & 3.570440 & -2.442744 & 0.464977 \\ \mathrm{H} & 3.305578 & -1.102390 & 1.639348 \\ \mathrm{H} & 0.867150 & 2.788013 & -0.962753 \\ \mathrm{H} & -3.860273 & 0.180192 & 0.673713 \\ \mathrm{H} & -3.148540 & -1.033678 & 1.775806 \\ \mathrm{H} & -2.959518 & 0.716813 & 2.136549 \\ \mathrm{H} & -1.243917 & 2.036279 & -1.044930 \\ \mathrm{H} & -0.228422 & 2.323572 & 0.961039\end{array}$


21

$\begin{array}{lrrr}\mathrm{P} & -0.374433 & 2.010238 & -0.092492 \\ \mathrm{O} & 2.665118 & -0.998117 & -0.397527 \\ \mathrm{O} & 1.572257 & 0.521912 & 0.922378 \\ \mathrm{O} & -2.423776 & 0.711478 & 0.517591 \\ \mathrm{O} & -1.893678 & -1.398768 & -0.218901 \\ \mathrm{~N} & 0.571545 & -0.382057 & -0.920906 \\ \mathrm{~N} & -0.531767 & 0.400492 & -0.631442 \\ \mathrm{C} & 3.853316 & -0.879935 & 0.436628 \\ \mathrm{C} & 1.639872 & -0.239299 & -0.039982 \\ \mathrm{C} & -3.123481 & -1.940490 & 0.362015 \\ \mathrm{C} & -1.711264 & -0.115729 & -0.046417 \\ \mathrm{H} & 4.583562 & -1.552128 & -0.020584 \\ \mathrm{H} & 4.215284 & 0.154529 & 0.427815 \\ \mathrm{H} & 3.621772 & -1.194249 & 1.460626 \\ \mathrm{H} & 0.839406 & 2.458854 & -0.642144 \\ \mathrm{H} & 0.732786 & -0.596675 & -1.912285 \\ \mathrm{H} & -3.989428 & -1.486220 & -0.131571 \\ \mathrm{H} & -3.140366 & -1.739489 & 1.438817 \\ \mathrm{H} & -3.074592 & -3.013267 & 0.162183 \\ \mathrm{H} & -1.378388 & 2.802280 & -0.683419 \\ \mathrm{H} & -0.382005 & 2.244440 & 1.292582\end{array}$

5

14

$\begin{array}{llll}\mathrm{P} & 0.000000 & -0.394300 & 0.000025\end{array}$

$\begin{array}{llll}\mathrm{O} & 1.711824 & -0.513390 & -0.000154\end{array}$

$\begin{array}{llll}\mathrm{O} & -1.711822 & -0.513390 & 0.000079\end{array}$

$\begin{array}{lrrr}\text { C } & 2.399699 & 0.726752 & 0.000027\end{array}$

$\begin{array}{llll}\text { C } & -2.399701 & 0.726752 & -0.000030\end{array}$

$\begin{array}{llll}\mathrm{H} & 3.479110 & 0.526614 & -0.000052\end{array}$

$\begin{array}{llll}\mathrm{H} & 2.153840 & 1.327952 & 0.897038\end{array}$

$\begin{array}{llll}\mathrm{H} & 2.153672 & 1.328471 & -0.896536\end{array}$

$\begin{array}{lllr}\mathrm{H} & 0.000140 & 0.422297 & 1.172085\end{array}$

$\begin{array}{llll}\mathrm{H} & 0.000001 & -1.803389 & -0.000132\end{array}$

$\mathrm{H} \quad-3.479111 \quad 0.526611 \quad-0.000218$ 


$\begin{array}{lrrr}\mathrm{H} & -2.153867 & 1.328317 & 0.896688 \\ \mathrm{H} & -2.153648 & 1.328104 & -0.896886 \\ \mathrm{H} & -0.000137 & 0.422747 & -1.171734\end{array}$

6

18

$\begin{array}{lrrr}\text { O } & 2.661297 & 0.211595 & 0.483916\end{array}$

$\begin{array}{llll}\mathrm{O} & 1.276270 & -0.632503 & -1.135771\end{array}$

$\begin{array}{llll}\text { O } & -2.662152 & 0.212374 & -0.483079\end{array}$

$\begin{array}{llll}\mathrm{O} & -1.276027 & -0.631268 & 1.135827\end{array}$

$\begin{array}{llll}\mathrm{N} & 0.656996 & 1.147810 & 0.203001\end{array}$

$\begin{array}{llll}\mathrm{N} & -0.656961 & 1.147925 & -0.204061\end{array}$

$\begin{array}{llll}\text { C } & 3.679340 & -0.723490 & 0.067173\end{array}$

$\begin{array}{llll}\text { C } & 1.507837 & 0.148139 & -0.229722\end{array}$

$\begin{array}{llll}\text { C } & -3.678692 & -0.724715 & -0.066996\end{array}$

$\begin{array}{llll}\text { C } & -1.508244 & 0.149147 & 0.229784\end{array}$

$\begin{array}{llll}\mathrm{H} & 4.530461 & -0.538657 & 0.729436\end{array}$

H $\quad 3.962576 \quad-0.544962 \quad-0.977852$

$\begin{array}{llll}\mathrm{H} & 3.325052 & -1.755828 & 0.179289\end{array}$

$\begin{array}{llrr}\mathrm{H} & 0.862466 & 1.619044 & 1.085365\end{array}$

$\mathrm{H} \quad-4.530152 \quad-0.540794 \quad-0.729069$

$\begin{array}{llll}\mathrm{H} & -3.962139 & -0.547574 & 0.978199\end{array}$

$\mathrm{H} \quad-3.322649 \quad-1.756364 \quad-0.179985$

$\mathrm{H} \quad-0.862420 \quad 1.618919-1.086545$

\section{7}

9

$\begin{array}{lrrr}\mathrm{P} & -0.990058 & 0.092382 & 0.000002 \\ \mathrm{O} & 0.420077 & -0.584225 & 0.000133 \\ \mathrm{C} & 1.664244 & 0.216973 & 0.000041 \\ \mathrm{H} & 2.466284 & -0.524580 & -0.011842 \\ \mathrm{H} & 1.706120 & 0.821680 & 0.913984 \\ \mathrm{H} & 1.695817 & 0.839220 & -0.902431 \\ \mathrm{H} & -1.922459 & -0.961169 & -0.008289 \\ \mathrm{H} & -1.223893 & 0.899452 & 1.139126 \\ \mathrm{H} & -1.217086 & 0.911633 & -1.131887\end{array}$


23

$\begin{array}{llll}\text { O } & 2.346308 & -0.785142 & 0.231068\end{array}$

$\begin{array}{llll}\mathrm{O} & 0.570458 & -1.867812 & -0.681057\end{array}$

$\begin{array}{llll}\mathrm{O} & 0.205253 & 2.672535 & -0.147865\end{array}$

$\begin{array}{llll}\mathrm{O} & -2.967045 & -0.213825 & -0.522663\end{array}$

$\begin{array}{llll}\mathrm{O} & -1.609335 & -0.204097 & 1.325226\end{array}$

$\begin{array}{llll}\mathrm{N} & 0.484950 & 0.419227 & -0.366682\end{array}$

$\begin{array}{llll}\mathrm{N} & -0.834206 & 0.368953 & -0.770006\end{array}$

$\begin{array}{llll}\text { C } & 3.045641 & -2.055046 & 0.295316\end{array}$

$\begin{array}{llll}\text { C } & 2.419599 & 1.910416 & 0.326375\end{array}$

$\begin{array}{llll}\text { C } & 1.117293 & -0.854606 & -0.296945\end{array}$

$\begin{array}{llll}\text { C } & 0.976869 & 1.734864 & -0.060539\end{array}$

$\begin{array}{llll}\text { C } & -4.076105 & -0.590682 & 0.322869\end{array}$

$\begin{array}{llll}\text { C } & -1.790641 & -0.045469 & 0.131229\end{array}$

$\begin{array}{llll}\mathrm{H} & 4.018673 & -1.820933 & 0.736014\end{array}$

H $\quad 3.165389 \quad-2.472715 \quad-0.711221$

$\begin{array}{llll}\mathrm{H} & 3.097737 & 1.471546 & -0.415123\end{array}$

$\begin{array}{llll}\mathrm{H} & 2.634235 & 1.424442 & 1.286255\end{array}$

$\begin{array}{llll}\mathrm{H} & 2.599417 & 2.986753 & 0.410325\end{array}$

$\begin{array}{llll}\mathrm{H} & 2.492257 & -2.759448 & 0.927189\end{array}$

$\mathrm{H} \quad-4.933453 \quad-0.683133 \quad-0.350417$

$\begin{array}{llll}\mathrm{H} & -4.264554 & 0.182552 & 1.078115\end{array}$

$\begin{array}{llll}\mathrm{H} & -3.874303 & -1.547913 & 0.819373\end{array}$

$\mathrm{H} \quad-1.011660 \quad 0.291456 \quad-1.771190$

9

11

$\begin{array}{rrrr}\mathrm{O} & 0.487475 & -0.769313 & 0.000000 \\ \mathrm{O} & -1.190991 & 0.753164 & 0.000000 \\ \mathrm{C} & 1.118610 & 1.504967 & 0.000000 \\ \mathrm{C} & 0.000000 & 0.497883 & 0.000000 \\ \mathrm{C} & -0.510906 & -1.814896 & 0.000000 \\ \mathrm{H} & 1.754823 & 1.363137 & 0.884300 \\ \mathrm{H} & 1.754823 & 1.363137 & -0.884300 \\ \mathrm{H} & 0.706123 & 2.517889 & 0.000000 \\ \mathrm{H} & 0.049529 & -2.754506 & 0.000000\end{array}$




$\begin{array}{rrrr}\mathrm{H} & -1.141699 & -1.744097 & 0.895008 \\ \mathrm{H} & -1.141699 & -1.744097 & -0.895008\end{array}$

\section{$\mathrm{AcOH}$}

\section{8}

$\begin{array}{rrrr}\mathrm{O} & 0.789921 & -1.041831 & -0.001061 \\ \mathrm{O} & 0.631318 & 1.213837 & 0.000173 \\ \mathrm{C} & 0.089310 & 0.123476 & -0.002281 \\ \mathrm{C} & -1.394000 & -0.121309 & -0.000517 \\ \mathrm{H} & 1.743193 & -0.803728 & 0.009888 \\ \mathrm{H} & -1.928910 & 0.830380 & -0.061575 \\ \mathrm{H} & -1.681682 & -0.649249 & 0.919763 \\ \mathrm{H} & -1.674375 & -0.766459 & -0.844177\end{array}$

$\mathrm{AcO}^{-}$

7

$\begin{array}{rrrr}\mathrm{O} & -0.771527 & -1.127061 & 0.002062 \\ \mathrm{O} & -0.756195 & 1.134930 & 0.002067 \\ \mathrm{C} & 1.354010 & -0.007539 & -0.004888 \\ \mathrm{C} & -0.186861 & -0.000026 & -0.010718 \\ \mathrm{H} & 1.759091 & 0.862382 & -0.538937 \\ \mathrm{H} & 1.754424 & -0.933512 & -0.437918 \\ \mathrm{H} & 1.705379 & 0.053565 & 1.037458\end{array}$

$E_{15-7}$

27

$\begin{array}{lrrr}\mathrm{P} & 0.493622 & -1.411610 & 0.758918 \\ \mathrm{O} & 2.081079 & -1.383758 & -0.685055 \\ \mathrm{O} & 1.341574 & 2.296071 & 0.457810 \\ \mathrm{O} & 1.075493 & 0.196692 & 1.063244 \\ \mathrm{O} & -2.532253 & -1.550016 & -0.900820 \\ \mathrm{O} & -2.447117 & 0.434076 & 0.230866 \\ \mathrm{~N} & -0.537953 & -0.364377 & -0.771920 \\ \mathrm{~N} & -0.069915 & 0.984688 & -0.809954\end{array}$




$\begin{array}{lrrr}\mathrm{C} & 3.446065 & -1.359530 & -0.171226 \\ \mathrm{C} & 1.041136 & 3.409380 & -0.434787 \\ \mathrm{C} & 0.739203 & 1.152009 & 0.194893 \\ \mathrm{C} & -3.855486 & 0.312588 & 0.600768 \\ \mathrm{C} & -1.970909 & -0.551583 & -0.510109 \\ \mathrm{H} & 4.134488 & -1.285646 & -1.021693 \\ \mathrm{H} & 3.657485 & -2.264449 & 0.414547 \\ \mathrm{H} & 3.529448 & -0.468606 & 0.457646 \\ \mathrm{H} & 1.966191 & -2.130036 & -1.343231 \\ \mathrm{H} & 1.611863 & 4.249558 & -0.030615 \\ \mathrm{H} & 1.450269 & -1.940199 & 1.653585 \\ \mathrm{H} & 1.366278 & 3.167664 & -1.453777 \\ \mathrm{H} & 0.142171 & -2.584050 & 0.036916 \\ \mathrm{H} & -4.471431 & 0.290814 & -0.305620 \\ \mathrm{H} & -4.065216 & 1.203434 & 1.197988 \\ \mathrm{H} & -4.005441 & -0.601234 & 1.187465 \\ \mathrm{H} & -0.636853 & -1.321127 & 1.607185 \\ \mathrm{H} & -0.344241 & -0.826641 & -1.696814 \\ \mathrm{H} & -0.034530 & 3.620791 & -0.419821\end{array}$

\section{$E_{3 d-17}$}

26

$\begin{array}{lrrr}\mathrm{P} & 0.388269 & 0.800641 & 1.347225 \\ \mathrm{O} & 2.619860 & -0.093980 & 0.325518 \\ \mathrm{O} & 1.878364 & -1.096482 & -1.605080 \\ \mathrm{O} & -2.701799 & -1.109920 & -1.208680 \\ \mathrm{O} & -1.407545 & -1.568533 & 0.625839 \\ \mathrm{O} & -0.599338 & 2.567483 & -0.475239 \\ \mathrm{~N} & 0.442164 & -0.032644 & -0.117362 \\ \mathrm{~N} & -0.693631 & -0.047007 & -0.974757 \\ \mathrm{C} & 3.980978 & -0.525267 & 0.062572 \\ \mathrm{C} & 1.679110 & -0.479260 & -0.579787 \\ \mathrm{C} & -2.430562 & -2.492087 & 1.051538 \\ \mathrm{C} & -1.773875 & 3.345361 & -0.216250 \\ \mathrm{C} & -1.658386 & -0.919901 & -0.572266 \\ \mathrm{H} & 4.573237 & -0.127337 & 0.891357 \\ \mathrm{H} & 4.323660 & -0.111875 & -0.893447 \\ \mathrm{H} & 4.029084 & -1.620729 & 0.041815 \\ \mathrm{H} & 1.107759 & 2.012298 & 1.422662\end{array}$




$\begin{array}{rrrr}\mathrm{H} & 0.890035 & -0.020753 & 2.383994 \\ \mathrm{H} & -3.389221 & -1.976142 & 1.194159 \\ \mathrm{H} & -2.558134 & -3.295636 & 0.314269 \\ \mathrm{H} & -2.500748 & 2.801913 & 0.412881 \\ \mathrm{H} & -2.273585 & 3.639459 & -1.153515 \\ \mathrm{H} & -2.075344 & -2.903838 & 2.002327 \\ \mathrm{H} & -1.462897 & 4.253660 & 0.315597 \\ \mathrm{H} & -0.961809 & 1.030083 & 1.649612 \\ \mathrm{H} & -0.865726 & 1.705210 & -0.918964\end{array}$

\section{$\mathrm{H}_{3} \mathrm{P}=\mathrm{O}$}

5

$\begin{array}{rrrr}\mathrm{P} & 0.386719 & 0.000015 & 0.000016 \\ \mathrm{O} & -1.109092 & 0.000004 & 0.000026 \\ \mathrm{H} & 1.024205 & -0.622222 & 1.111911 \\ \mathrm{H} & 1.024090 & 1.274116 & -0.017427 \\ \mathrm{H} & 1.023664 & -0.652148 & -1.094935\end{array}$

\section{$\mathrm{MeOH}$}

6

$\begin{array}{rrrr}\mathrm{O} & -0.047617 & -0.762170 & 0.000000 \\ \mathrm{C} & -0.047617 & 0.668053 & 0.000000 \\ \mathrm{H} & 0.876630 & -1.064839 & 0.000000 \\ \mathrm{H} & 0.443337 & 1.082082 & 0.896968 \\ \mathrm{H} & 0.443337 & 1.082082 & -0.896968 \\ \mathrm{H} & -1.096661 & 0.989719 & 0.000000\end{array}$

\section{$\mathrm{MeO}^{-}$}

\section{5}

$\begin{array}{llll}\text { O } & 0.000000 & -0.000000 & 0.812540\end{array}$

$\begin{array}{llll}\text { C } & -0.000000 & 0.000000 & -0.569393\end{array}$

$\begin{array}{llll}\mathrm{H} & 0.887917 & -0.512639 & -1.027989\end{array}$

$\begin{array}{llll}\mathrm{H} & 0.000000 & 1.025278 & -1.027989\end{array}$

$\mathrm{H} \quad-0.887917 \quad-0.512639 \quad-1.027989$ 


\section{$\mathrm{PH}_{3}$}

4

$\begin{array}{rrrr}\mathrm{P} & -0.000013 & 0.000001 & -0.131014 \\ \mathrm{H} & 0.597375 & -1.033914 & 0.655019 \\ \mathrm{H} & 0.597041 & 1.034094 & 0.655033 \\ \mathrm{H} & -1.194216 & -0.000199 & 0.655158\end{array}$

$T_{15-7}$

27

$\begin{array}{lrrr}\mathrm{P} & 0.888759 & -1.601372 & 0.580551 \\ \mathrm{O} & 2.083432 & -0.865224 & -0.618524 \\ \mathrm{O} & 0.625359 & 0.265161 & 1.281572 \\ \mathrm{O} & 0.607218 & 2.363023 & 0.574882 \\ \mathrm{O} & -2.334345 & -1.852130 & -0.660457 \\ \mathrm{O} & -2.203178 & 0.299253 & 0.099790 \\ \mathrm{~N} & 0.224321 & 0.648050 & -0.962983 \\ \mathrm{~N} & -0.339963 & -0.684212 & -0.834395 \\ \mathrm{C} & 3.418405 & -0.543054 & -0.138499 \\ \mathrm{C} & 0.446413 & 1.090631 & 0.331057 \\ \mathrm{C} & 0.340427 & 3.335588 & -0.492730 \\ \mathrm{C} & -3.593737 & 0.259949 & 0.558950 \\ \mathrm{C} & -1.762759 & -0.807062 & -0.467813 \\ \mathrm{H} & 3.946772 & -1.477219 & 0.086236 \\ \mathrm{H} & 3.927584 & -0.018617 & -0.955702 \\ \mathrm{H} & 3.374785 & 0.097127 & 0.753914 \\ \mathrm{H} & 1.902369 & -1.758152 & 1.554379 \\ \mathrm{H} & 1.402770 & 0.102340 & -1.048595 \\ \mathrm{H} & 1.172725 & 3.327227 & -1.205289 \\ \mathrm{H} & 0.941118 & -2.817504 & -0.141340 \\ \mathrm{H} & 0.282101 & 4.295978 & 0.025624 \\ \mathrm{H} & -4.252556 & 0.094618 & -0.301006 \\ \mathrm{H} & -3.767065 & 1.240804 & 1.007967 \\ \mathrm{H} & -3.710888 & -0.542052 & 1.296505 \\ \mathrm{H} & -0.601341 & 3.090441 & -0.993630 \\ \mathrm{H} & -0.246353 & -1.879703 & 1.389782 \\ \mathrm{H} & -0.214303 & -1.178550 & -1.759367\end{array}$




\section{$T_{16-8}$}

28

$\begin{array}{llll}\mathrm{P} & 1.116144 & -1.361292 & -1.462509\end{array}$

$\begin{array}{llrr}\text { O } & 2.820574 & 0.782972 & 0.814529\end{array}$

$\begin{array}{llll}\text { O } & 2.404747 & -1.080805 & -0.611996\end{array}$

$\begin{array}{llll}\mathrm{O} & 0.896279 & 1.822155 & -1.218780\end{array}$

$\begin{array}{llll}\mathrm{O} & -2.827382 & -0.846117 & 1.616954\end{array}$

O $\quad-2.031902 \quad-0.923173 \quad-0.528336$

$\begin{array}{llll}\mathrm{O} & -0.798025 & 2.166871 & 0.275255\end{array}$

$\begin{array}{llll}\mathrm{N} & 0.301292 & 0.139777 & 0.212715\end{array}$

$\begin{array}{llll}\mathrm{N} & -0.666985 & -0.248098 & 1.154695\end{array}$

C $\quad 2.156953 \quad-0.221460 \quad 0.763773$

$\begin{array}{llll}\text { C } & 1.982169 & -1.248243 & 1.849314\end{array}$

$\begin{array}{llll}\text { C } & 0.177065 & 1.421476 & -0.306762\end{array}$

$\begin{array}{llll}\text { C } & -3.322577 & -1.424537 & -0.953783\end{array}$

$\begin{array}{llll}\text { C } & -1.924587 & -0.677319 & 0.802396\end{array}$

$\begin{array}{llll}\text { C } & -0.982612 & 3.485496 & -0.291964\end{array}$

$\begin{array}{llll}\mathrm{H} & 2.954760 & -1.744527 & 1.977157\end{array}$

$\begin{array}{llll}\mathrm{H} & 1.719437 & -0.745341 & 2.788691\end{array}$

H $\quad 1.659433 \quad-2.223491 \quad-2.453502$

$\mathrm{H} \quad 1.224122 \quad-2.001081 \quad 1.613632$

$\begin{array}{llll}\mathrm{H} & 0.559109 & -0.320994 & -2.230587\end{array}$

H $\quad 0.105183 \quad-2.160788 \quad-0.896430$

$\mathrm{H} \quad-4.111280 \quad-0.700140 \quad-0.715938$

$\mathrm{H} \quad-3.542087 \quad-2.383131 \quad-0.467072$

$\mathrm{H} \quad-3.236432 \quad-1.554796 \quad-2.036841$

$\begin{array}{llll}\mathrm{H} & -1.810171 & 3.928082 & 0.271047\end{array}$

$\begin{array}{llll}\mathrm{H} & -1.238029 & 3.412033 & -1.356799\end{array}$

$\begin{array}{llll}\mathrm{H} & -0.627356 & 0.198163 & 2.086692\end{array}$

$\begin{array}{llll}\mathrm{H} & -0.071798 & 4.085937 & -0.173136\end{array}$

\section{$T_{17-5}$}

32

$\begin{array}{lrrr}\mathrm{P} & -1.499736 & -1.065787 & -0.374233 \\ \mathrm{O} & 2.969537 & -0.035571 & -1.871463 \\ \mathrm{O} & 2.190520 & -0.754375 & 0.158919 \\ \mathrm{O} & 0.572979 & 2.506432 & 0.230358\end{array}$




$\begin{array}{lrrr}\mathrm{O} & -2.556540 & -0.267626 & -1.366312 \\ \mathrm{O} & -1.242915 & 1.660716 & 1.330623 \\ \mathrm{O} & -0.062242 & -2.210972 & 0.839196 \\ \mathrm{~N} & 0.814499 & 0.403486 & -1.233023 \\ \mathrm{~N} & -0.184447 & 0.403487 & -0.270541 \\ \mathrm{C} & 3.507513 & -1.314253 & 0.392400 \\ \mathrm{C} & 2.065273 & -0.111517 & -1.054557 \\ \mathrm{C} & 0.419952 & 3.697425 & 1.028292 \\ \mathrm{C} & -3.902694 & -0.159023 & -0.890957 \\ \mathrm{C} & -0.400899 & -2.296932 & 2.237420 \\ \mathrm{C} & -0.352751 & 1.529737 & 0.493258 \\ \mathrm{H} & 4.265237 & -0.523481 & 0.355403 \\ \mathrm{H} & 3.737345 & -2.081948 & -0.355920 \\ \mathrm{H} & 3.462504 & -1.753025 & 1.393927 \\ \mathrm{H} & 1.223662 & 4.367698 & 0.705602 \\ \mathrm{H} & 0.766819 & -1.678609 & 0.732892 \\ \mathrm{H} & 0.671452 & 0.926996 & -2.099781 \\ \mathrm{H} & 0.520751 & 3.467585 & 2.096905 \\ \mathrm{H} & 0.365736 & -2.875217 & 2.771012 \\ \mathrm{H} & -4.490401 & 0.318625 & -1.684404 \\ \mathrm{H} & -4.332596 & -1.153006 & -0.678629 \\ \mathrm{H} & -3.953906 & 0.461979 & 0.017923 \\ \mathrm{H} & -2.228971 & -2.316059 & -0.301466 \\ \mathrm{H} & -1.907403 & -0.612233 & 0.908300 \\ \mathrm{H} & -1.359261 & -2.824902 & 2.303300 \\ \mathrm{H} & -0.587580 & -1.513100 & -1.371659 \\ \mathrm{H} & -0.558035 & 4.163428 & 0.851478 \\ \mathrm{H} & -0.498750 & -1.298183 & 2.687856\end{array}$

\section{$T_{20-10}$}

21

P $\quad-0.636811 \quad-0.394231 \quad-0.068030$

$\begin{array}{llll}\text { O } & 3.340096 & -0.226974 & -0.792000\end{array}$

O $\quad 1.119959 \quad-0.153701 \quad-0.781211$

$\begin{array}{lllr}\mathrm{O} & -2.217170 & -0.571219 & 0.546490\end{array}$

$\begin{array}{llll}\mathrm{O} & -0.955428 & 1.788822 & -0.598801\end{array}$

$\begin{array}{llll}\text { C } & 2.269354 & -0.369252 & -0.178565\end{array}$

$\begin{array}{llll}\text { C } & 2.267180 & -0.788525 & 1.282122\end{array}$

C $\quad-3.089636 \quad-1.372428 \quad-0.240502$ 


$\begin{array}{lrrr}\mathrm{C} & -0.564435 & 2.565730 & 0.484617 \\ \mathrm{H} & 3.278293 & -1.084013 & 1.580383 \\ \mathrm{H} & 1.940216 & 0.049955 & 1.913876 \\ \mathrm{H} & 1.571079 & -1.620437 & 1.459040 \\ \mathrm{H} & 0.512067 & 2.437250 & 0.759583 \\ \mathrm{H} & -4.059229 & -1.426662 & 0.273419 \\ \mathrm{H} & -3.247593 & -0.936251 & -1.243994 \\ \mathrm{H} & -2.700078 & -2.400899 & -0.364403 \\ \mathrm{H} & -1.146364 & 2.362105 & 1.417326 \\ \mathrm{H} & -0.992390 & -0.467882 & -1.440235 \\ \mathrm{H} & -0.697144 & 3.647960 & 0.273130 \\ \mathrm{H} & -0.278772 & -1.762261 & 0.163814 \\ \mathrm{H} & -0.222366 & 0.206027 & 1.146663\end{array}$

\section{$T_{\text {21-9Ret }}$}

16

$\begin{array}{lrrr}\mathrm{P} & 1.617852 & -0.327601 & 0.089997 \\ \mathrm{O} & 0.389727 & -1.326949 & 0.149515 \\ \mathrm{O} & 0.091465 & 0.830563 & -0.547331 \\ \mathrm{O} & -1.587394 & -0.849480 & -1.149031 \\ \mathrm{C} & -1.603449 & -0.178734 & 1.179223 \\ \mathrm{C} & -0.982156 & -0.461145 & -0.168457 \\ \mathrm{C} & -0.119033 & 2.151133 & -0.045559 \\ \mathrm{H} & 2.602943 & -1.265460 & 0.526195 \\ \mathrm{H} & 2.234298 & 0.077307 & -1.115142 \\ \mathrm{H} & 1.828010 & 0.663122 & 1.081047 \\ \mathrm{H} & 0.607497 & 2.822500 & -0.527191 \\ \mathrm{H} & 0.009293 & 2.209367 & 1.048998 \\ \mathrm{H} & -2.420372 & 0.545163 & 1.058578 \\ \mathrm{H} & -2.031182 & -1.119933 & 1.550544 \\ \mathrm{H} & -1.133154 & 2.485320 & -0.308674 \\ \mathrm{H} & -0.887672 & 0.196033 & 1.919223\end{array}$

$T_{22-23}$

18

$\begin{array}{llll}\mathrm{P} & 0.963759 & -1.467851 & 0.005576\end{array}$ 


$\begin{array}{lrrr}\mathrm{O} & 1.050390 & -0.009650 & -1.065366 \\ \mathrm{O} & 1.024943 & -0.006437 & 1.074150 \\ \mathrm{O} & -1.075666 & -1.100536 & -0.018750 \\ \mathrm{O} & -0.847772 & 1.149922 & -0.020480 \\ \mathrm{C} & 1.442946 & 2.174254 & 0.005940 \\ \mathrm{C} & 0.871967 & 0.791779 & 0.001207 \\ \mathrm{C} & -3.053673 & 0.242494 & 0.010641 \\ \mathrm{C} & -1.558717 & 0.075933 & -0.019337 \\ \mathrm{H} & 2.538488 & 2.093331 & 0.018955 \\ \mathrm{H} & 2.365513 & -1.681364 & 0.022193 \\ \mathrm{H} & 1.131611 & 2.715875 & -0.894064 \\ \mathrm{H} & 1.110512 & 2.718469 & 0.896772 \\ \mathrm{H} & 0.631467 & -2.319708 & -1.076168 \\ \mathrm{H} & 0.606151 & -2.316278 & 1.081947 \\ \mathrm{H} & -3.536719 & -0.577719 & -0.532866 \\ \mathrm{H} & -3.386303 & 0.200190 & 1.059295 \\ \mathrm{H} & -3.347401 & 1.211806 & -0.406837\end{array}$

$T_{23-12}$

18

$\begin{array}{lrrr}\mathrm{P} & 1.857444 & -1.075620 & -0.069548 \\ \mathrm{O} & 1.096956 & -0.032014 & -0.968617 \\ \mathrm{O} & 0.771833 & 0.433252 & 1.302655 \\ \mathrm{O} & -1.162677 & -1.497220 & 0.134671 \\ \mathrm{O} & -1.080199 & 0.773174 & -0.120572 \\ \mathrm{C} & 0.713922 & 2.338209 & -0.213845 \\ \mathrm{C} & 0.367804 & 0.922075 & 0.199545 \\ \mathrm{C} & -3.211352 & -0.232941 & -0.109309 \\ \mathrm{C} & -1.716102 & -0.418035 & -0.011787 \\ \mathrm{H} & 2.964458 & -0.603260 & 0.662274 \\ \mathrm{H} & 2.444653 & -1.899375 & -1.058859 \\ \mathrm{H} & 1.797593 & 2.479015 & -0.127099 \\ \mathrm{H} & 1.139346 & -1.974616 & 0.734701 \\ \mathrm{H} & 0.391535 & 2.551362 & -1.239251 \\ \mathrm{H} & 0.210048 & 3.032850 & 0.472218 \\ \mathrm{H} & -3.703859 & -1.207869 & -0.177970 \\ \mathrm{H} & -3.570914 & 0.297774 & 0.784582 \\ \mathrm{H} & -3.467457 & 0.385037 & -0.980108\end{array}$




\section{$T_{3 d-17}$}

26

$\begin{array}{llll}\mathrm{P} & 0.783307 & 0.954154 & 1.342113\end{array}$

$\begin{array}{llll}\text { O } & 2.297762 & -1.089829 & 0.240447\end{array}$

$\begin{array}{llll}\mathrm{O} & 0.848710 & -1.784523 & -1.389596\end{array}$

$\begin{array}{lllr}\mathrm{O} & 0.039686 & 2.560293 & 0.026860\end{array}$

$\begin{array}{llll}\text { O } & -3.026294 & 0.276158 & -1.025083\end{array}$

$\begin{array}{llll}\mathrm{O} & -2.000822 & -1.050566 & 0.528730\end{array}$

$\begin{array}{lrrr}\mathrm{N} & 0.305651 & -0.054824 & 0.048964\end{array}$

$\begin{array}{llll}\mathrm{N} & -0.764903 & 0.415012 & -0.769646\end{array}$

$\begin{array}{llll}\text { C } & 3.218489 & -2.153291 & -0.117770\end{array}$

$\begin{array}{llll}\text { C } & 1.138133 & -1.059996 & -0.458126\end{array}$

$\begin{array}{llll}\text { C } & 1.017530 & 3.158031 & -0.834519\end{array}$

$\begin{array}{llll}\text { C } & -3.294372 & -1.616185 & 0.830682\end{array}$

$\begin{array}{llll}\text { C } & -1.998768 & -0.095587 & -0.450293\end{array}$

$\begin{array}{llll}\mathrm{H} & 4.076451 & -2.024111 & 0.548171\end{array}$

$\begin{array}{llll}\mathrm{H} & 3.522195 & -2.049547 & -1.166410\end{array}$

$\begin{array}{lllr}\mathrm{H} & 2.746834 & -3.130508 & 0.042659\end{array}$

$\begin{array}{llll}\mathrm{H} & 2.000758 & 3.185661 & -0.332504\end{array}$

$\mathrm{H} \quad 1.896801 \quad 1.825759 \quad 1.278952$

$\begin{array}{lllr}\mathrm{H} & 1.278320 & -0.028632 & 2.237699\end{array}$

$\begin{array}{llll}\mathrm{H} & 1.117674 & 2.595721 & -1.776567\end{array}$

$\begin{array}{llll}\mathrm{H} & 0.720926 & 4.192014 & -1.061362\end{array}$

$\mathrm{H} \quad-3.986403 \quad-0.840216 \quad 1.183128$

$\begin{array}{llll}\mathrm{H} & -3.718834 & -2.106695 & -0.055061\end{array}$

$\begin{array}{llll}\mathrm{H} & -3.111939 & -2.351214 & 1.621545\end{array}$

$\begin{array}{llll}\mathrm{H} & -0.515687 & 1.762611 & -0.539842\end{array}$

$\mathrm{H} \quad-0.320350 \quad 1.445444 \quad 2.061955$

$\mathbf{T}_{4-7}$

27

$\begin{array}{lllr}\mathrm{P} & 0.390032 & 1.250804 & 1.087102\end{array}$

$\begin{array}{llll}\text { O } & 1.987169 & -2.152580 & -0.277803\end{array}$

$\begin{array}{llll}\mathrm{O} & 1.781821 & 0.010351 & 0.418744\end{array}$

$\begin{array}{llll}\text { O } & 0.520281 & 2.084906 & -0.533629\end{array}$

$\begin{array}{llll}\mathrm{O} & -2.736257 & 1.078665 & 0.054627\end{array}$

O $\quad-2.549506 \quad-1.188870 \quad-0.179235$ 


$\begin{array}{lrrr}\mathrm{N} & -0.681674 & 0.123143 & -0.293807 \\ \mathrm{~N} & -0.001051 & -1.103667 & -0.405538 \\ \mathrm{C} & 3.394303 & -2.131489 & 0.123644 \\ \mathrm{C} & 1.770662 & 2.531648 & -1.113169 \\ \mathrm{C} & 1.292514 & -1.062782 & -0.071228 \\ \mathrm{C} & -3.993973 & -1.325091 & -0.056728 \\ \mathrm{C} & -2.092290 & 0.064883 & -0.112876 \\ \mathrm{H} & 3.923325 & -1.344047 & -0.423964 \\ \mathrm{H} & 3.769056 & -3.119205 & -0.153340 \\ \mathrm{H} & 3.467873 & -1.974058 & 1.205113 \\ \mathrm{H} & 2.356835 & 1.683782 & -1.487011 \\ \mathrm{H} & 2.335872 & 3.077631 & -0.347451 \\ \mathrm{H} & 1.511196 & 3.215489 & -1.928925 \\ \mathrm{H} & 1.438610 & 2.006450 & 1.658620 \\ \mathrm{H} & 0.207749 & 0.264911 & 2.098537 \\ \mathrm{H} & -4.488140 & -0.763759 & -0.857720 \\ \mathrm{H} & -4.319161 & -0.954970 & 0.922022 \\ \mathrm{H} & -4.186118 & -2.396546 & -0.153110 \\ \mathrm{H} & -0.752055 & 2.033556 & 1.366456 \\ \mathrm{H} & -0.451335 & -1.905678 & -0.858768 \\ \mathrm{H} & -0.140471 & 1.155258 & -0.931050\end{array}$

\section{$\mathbf{T}_{\text {5-20 }}$}

21

$\begin{array}{lrrr}\mathrm{P} & -0.999026 & -0.312105 & -0.225264 \\ \mathrm{O} & 4.730724 & -0.025040 & -0.345146 \\ \mathrm{O} & 2.589652 & 0.406706 & -0.931469 \\ \mathrm{O} & -2.258479 & -1.339245 & 0.078207 \\ \mathrm{O} & -1.978996 & 1.101150 & -0.521009 \\ \mathrm{C} & 3.480040 & 0.022625 & -0.112798 \\ \mathrm{C} & 2.998939 & -0.397655 & 1.288846 \\ \mathrm{C} & -3.664138 & -1.028047 & -0.029749 \\ \mathrm{C} & -2.416062 & 1.871674 & 0.593232 \\ \mathrm{H} & 3.759567 & -0.980199 & 1.823732 \\ \mathrm{H} & 2.781353 & 0.507356 & 1.877128 \\ \mathrm{H} & 2.065344 & -0.971918 & 1.219330 \\ \mathrm{H} & -4.158743 & -1.951050 & -0.358462 \\ \mathrm{H} & -4.059892 & -0.736789 & 0.953860 \\ \mathrm{H} & -3.845200 & -0.228444 & -0.756123\end{array}$




$\begin{array}{rrrr}\mathrm{H} & -3.369571 & 1.505145 & 1.009730 \\ \mathrm{H} & -2.553252 & 2.910769 & 0.262327 \\ \mathrm{H} & -1.659236 & 1.862309 & 1.403070 \\ \mathrm{H} & -0.522390 & -0.123345 & -1.547804 \\ \mathrm{H} & -0.333843 & 0.411673 & 0.815393 \\ \mathrm{H} & -0.174623 & -1.484075 & -0.005072\end{array}$

$T_{7-9 I n v}$

16

$\begin{array}{llll}\mathrm{P} & -3.294723 & -0.206516 & 0.017319\end{array}$

$\begin{array}{llll}\mathrm{O} & 2.177885 & 1.258909 & 0.002044\end{array}$

$\begin{array}{llll}\text { O } & 1.593824 & -0.926603 & -0.034624\end{array}$

$\begin{array}{llll}\mathrm{O} & -2.053620 & 0.708428 & -0.015859\end{array}$

$\begin{array}{llll}\text { C } & 3.928599 & -0.377052 & 0.029849\end{array}$

$\begin{array}{llll}\text { C } & 2.447050 & 0.028013 & -0.007077\end{array}$

$\begin{array}{llll}\text { C } & -0.542710 & 0.007372 & -0.026375\end{array}$

$\begin{array}{llll}\mathrm{H} & 4.481074 & 0.154378 & -0.757589\end{array}$

$\mathrm{H} \quad 4.364116 \quad-0.069040 \quad 0.991959$

$\begin{array}{llll}\mathrm{H} & 4.063723 & -1.458513 & -0.091480\end{array}$

$\begin{array}{llll}\mathrm{H} & 0.091987 & 0.894653 & -0.019773\end{array}$

$\begin{array}{llll}\mathrm{H} & -4.468085 & 0.575649 & 0.033507\end{array}$

$\begin{array}{llll}\mathrm{H} & -3.411933 & -1.077042 & -1.094213\end{array}$

$\begin{array}{llll}\mathrm{H} & -3.365192 & -1.054692 & 1.149852\end{array}$

$\mathrm{H} \quad-0.542366 \quad-0.563531 \quad-0.952651$

H $\quad-0.534834 \quad-0.579990 \quad 0.889730$

\section{T7-9ret}

16

$\begin{array}{lrrr}\mathrm{P} & -1.676739 & -0.956474 & 0.231516 \\ \mathrm{O} & 1.762469 & 0.656786 & 1.144484 \\ \mathrm{O} & 0.490289 & 0.078137 & -0.630451 \\ \mathrm{O} & -2.111352 & 0.407187 & -0.310296 \\ \mathrm{C} & 2.663419 & -0.885360 & -0.483523 \\ \mathrm{C} & 1.575453 & 0.022521 & 0.086818 \\ \mathrm{C} & -0.618259 & 1.954093 & -0.245314 \\ \mathrm{H} & 3.371575 & -1.170185 & 0.303133\end{array}$




$\begin{array}{rrrr}\mathrm{H} & 3.208003 & -0.331869 & -1.263590 \\ \mathrm{H} & 2.232124 & -1.778804 & -0.951945 \\ \mathrm{H} & 0.226794 & 1.990259 & 0.439609 \\ \mathrm{H} & -2.820457 & -1.637926 & 0.729297 \\ \mathrm{H} & -1.563509 & 2.330237 & 0.133756 \\ \mathrm{H} & -1.123947 & -1.880877 & -0.683322 \\ \mathrm{H} & -0.807766 & -0.957218 & 1.346608 \\ \mathrm{H} & -0.426667 & 2.099078 & -1.304068\end{array}$

\subsection{Coordinates in the Gas Phase}

All structures were fully optimized at the bp86/6-311++G(3df,3pd) level of theory.

10

16

$\begin{array}{llll}\mathrm{P} & 0.000000 & 0.927335 & -0.000000\end{array}$

$\begin{array}{llll}\mathrm{O} & 1.273677 & -0.380605 & 0.000000\end{array}$

$\begin{array}{llll}\mathrm{O} & -0.997376 & 2.294896 & -0.000000\end{array}$

$\begin{array}{llll}\text { O } & -0.363214 & -1.931468 & 0.000000\end{array}$

$\begin{array}{llll}\text { C } & 1.947989 & -2.665838 & 0.000000\end{array}$

$\begin{array}{llll}\text { C } & 0.829841 & -1.647151 & 0.000000\end{array}$

$\begin{array}{llll}\text { C } & -2.400099 & 2.049287 & -0.000000\end{array}$

$\mathrm{H} \quad 2.585029 \quad-2.522511 \quad 0.883575$

$\mathrm{H} \quad 2.585029 \quad-2.522511-0.883575$

$\begin{array}{llll}\mathrm{H} & 1.531049 & -3.677371 & 0.000000\end{array}$

$\begin{array}{llll}\mathrm{H} & 1.114577 & 1.778898 & -0.000000\end{array}$

$\mathrm{H} \quad-2.913319 \quad 3.018765-0.000000$

$\mathrm{H} \quad-2.711192 \quad 1.481982 \quad 0.896639$

$\begin{array}{llll}\mathrm{H} & -2.711192 & 1.481982 & -0.896639\end{array}$

$\begin{array}{llll}\mathrm{H} & -0.525534 & 0.385186 & 1.193293\end{array}$

$\mathrm{H} \quad-0.525534 \quad 0.385186 \quad-1.193293$

\section{1}

11

$\begin{array}{llll}\mathrm{P} & 1.542632 & 0.376761 & -0.000000\end{array}$

$\begin{array}{lrrr}\text { O } & 0.000000 & 0.823823 & -0.000000\end{array}$

$\begin{array}{llll}\text { O } & -0.289248 & -1.415583 & 0.000000\end{array}$

$\begin{array}{llll}\text { C } & -2.295159 & 0.001249 & -0.000000\end{array}$ 


$\begin{array}{rrrr}\mathrm{C} & -0.861197 & -0.357895 & 0.000000 \\ \mathrm{H} & 2.219233 & 1.608961 & -0.000000 \\ \mathrm{H} & 1.918520 & -0.352353 & 1.144621 \\ \mathrm{H} & 1.918520 & -0.352353 & -1.144621 \\ \mathrm{H} & -2.897092 & -0.911839 & 0.000000 \\ \mathrm{H} & -2.523268 & 0.615064 & 0.883830 \\ \mathrm{H} & -2.523268 & 0.615064 & -0.883830\end{array}$

12

13

$\begin{array}{lrrr}\mathrm{O} & 1.276567 & 1.160555 & 0.697925 \\ \mathrm{O} & 0.000010 & -0.615370 & -0.000067 \\ \mathrm{O} & -1.276628 & 1.160535 & -0.697911 \\ \mathrm{C} & 2.353991 & -0.744945 & -0.349162 \\ \mathrm{C} & 1.211471 & 0.085098 & 0.168150 \\ \mathrm{C} & -2.353949 & -0.744976 & 0.349205 \\ \mathrm{C} & -1.211461 & 0.085037 & -0.168207 \\ \mathrm{H} & 3.303599 & -0.277062 & -0.074381 \\ \mathrm{H} & 2.298326 & -1.765629 & 0.051330 \\ \mathrm{H} & 2.279152 & -0.820893 & -1.443534 \\ \mathrm{H} & -3.303592 & -0.277841 & 0.073274 \\ \mathrm{H} & -2.297552 & -1.766150 & -0.049913 \\ \mathrm{H} & -2.279836 & -0.819472 & 1.443739\end{array}$

13

20

$\begin{array}{lrrr}\mathrm{P} & 0.029849 & 2.158491 & -0.074096 \\ \mathrm{O} & 2.727998 & 0.354178 & -0.846953 \\ \mathrm{O} & 1.641766 & -1.150086 & 0.492933 \\ \mathrm{O} & -2.809765 & 0.180661 & 0.953716 \\ \mathrm{O} & -1.600378 & -1.061480 & -0.538994 \\ \mathrm{~N} & 0.483597 & 0.586303 & -0.389752 \\ \mathrm{~N} & -0.573654 & 0.582580 & 0.707469 \\ \mathrm{C} & 2.871280 & -1.904824 & 0.600280 \\ \mathrm{C} & 1.734120 & -0.047778 & -0.272442 \\ \mathrm{C} & -2.797432 & -1.822803 & -0.805643 \\ \mathrm{C} & -1.757708 & -0.083586 & 0.393350\end{array}$




$\begin{array}{rrrr}\mathrm{H} & 3.660611 & -1.289732 & 1.049909 \\ \mathrm{H} & 3.196866 & -2.246184 & -0.390206 \\ \mathrm{H} & 2.629023 & -2.755180 & 1.244026 \\ \mathrm{H} & 0.925258 & 2.932582 & -0.888984 \\ \mathrm{H} & 0.237293 & 2.814899 & 1.162063 \\ \mathrm{H} & -3.602673 & -1.167826 & -1.162244 \\ \mathrm{H} & -3.133613 & -2.340463 & 0.102033 \\ \mathrm{H} & -2.515698 & -2.544235 & -1.579002 \\ \mathrm{H} & -1.192935 & 2.604344 & -0.629052\end{array}$

\section{$14 a$}

20

$\begin{array}{lrrr}\mathrm{P} & -0.167783 & 2.029805 & 0.000422 \\ \mathrm{O} & 2.662010 & 0.839845 & -0.000716 \\ \mathrm{O} & 1.898988 & -1.306129 & 0.000355 \\ \mathrm{O} & -2.901860 & -0.740802 & -0.000390 \\ \mathrm{O} & -1.856899 & 1.241763 & -0.000945 \\ \mathrm{~N} & 0.382180 & 0.381057 & 0.000369 \\ \mathrm{~N} & -0.583771 & -0.654057 & 0.000498 \\ \mathrm{C} & 3.279852 & -1.731703 & -0.000216 \\ \mathrm{C} & 1.742982 & 0.036521 & 0.000046 \\ \mathrm{C} & -2.779961 & -2.180791 & 0.000232 \\ \mathrm{C} & -1.745062 & -0.069472 & -0.000307 \\ \mathrm{H} & 3.798719 & -1.362786 & 0.893450 \\ \mathrm{H} & 3.797876 & -1.363130 & -0.894513 \\ \mathrm{H} & 3.238459 & -2.825052 & 0.000015 \\ \mathrm{H} & 1.166427 & 2.526528 & 0.001160 \\ \mathrm{H} & -3.808158 & -2.554453 & 0.000000 \\ \mathrm{H} & -2.241835 & -2.519348 & 0.895267 \\ \mathrm{H} & -2.241169 & -2.520063 & -0.894132 \\ \mathrm{H} & -0.593986 & 2.738063 & 1.152869 \\ \mathrm{H} & -0.593229 & 2.739416 & -1.151481\end{array}$

$14 b$

20

$\begin{array}{llll}\mathrm{P} & -0.167783 & 2.029805 & 0.000422\end{array}$ 


$\begin{array}{lrrr}\mathrm{O} & 2.662010 & 0.839845 & -0.000716 \\ \mathrm{O} & 1.898988 & -1.306129 & 0.000355 \\ \mathrm{O} & -2.901860 & -0.740802 & -0.000390 \\ \mathrm{O} & -1.856899 & 1.241763 & -0.000945 \\ \mathrm{~N} & 0.382180 & 0.381057 & 0.000369 \\ \mathrm{~N} & -0.583771 & -0.654057 & 0.000498 \\ \mathrm{C} & 3.279852 & -1.731703 & -0.000216 \\ \mathrm{C} & 1.742982 & 0.036521 & 0.000046 \\ \mathrm{C} & -2.779961 & -2.180791 & 0.000232 \\ \mathrm{C} & -1.745062 & -0.069472 & -0.000307 \\ \mathrm{H} & 3.798719 & -1.362786 & 0.893450 \\ \mathrm{H} & 3.797876 & -1.363130 & -0.894513 \\ \mathrm{H} & 3.238459 & -2.825052 & 0.000015 \\ \mathrm{H} & 1.166427 & 2.526528 & 0.001160 \\ \mathrm{H} & -3.808158 & -2.554453 & 0.000000 \\ \mathrm{H} & -2.241835 & -2.519348 & 0.895267 \\ \mathrm{H} & -2.241169 & -2.520063 & -0.894132 \\ \mathrm{H} & -0.593986 & 2.738063 & 1.152869 \\ \mathrm{H} & -0.593229 & 2.739416 & -1.151481\end{array}$

15

21

$\begin{array}{lrrr}\mathrm{P} & 0.095638 & 2.131660 & -0.303299 \\ \mathrm{O} & 2.646803 & -0.594030 & -0.383738 \\ \mathrm{O} & 1.300335 & 1.111831 & -0.704466 \\ \mathrm{O} & -2.737952 & 0.346219 & 0.789323 \\ \mathrm{O} & -1.541565 & -1.219720 & -0.380760 \\ \mathrm{~N} & 0.704244 & -0.548558 & 0.871644 \\ \mathrm{~N} & -0.438138 & 0.310677 & 0.939728 \\ \mathrm{C} & 3.023339 & -1.869470 & 0.240872 \\ \mathrm{C} & 1.516415 & -0.071723 & -0.009752 \\ \mathrm{C} & -2.772374 & -1.790742 & -0.943031 \\ \mathrm{C} & -1.721511 & -0.217235 & 0.458801 \\ \mathrm{H} & 3.964062 & -2.138696 & -0.242893 \\ \mathrm{H} & 3.155505 & -1.719703 & 1.317755 \\ \mathrm{H} & 2.242699 & -2.612585 & 0.046727 \\ \mathrm{H} & 0.566572 & 3.143661 & -1.174488 \\ \mathrm{H} & 0.128766 & 2.793380 & 0.942150 \\ \mathrm{H} & -3.365665 & -2.231578 & -0.135109\end{array}$




$\begin{array}{lrrr}\mathrm{H} & -3.344889 & -1.007625 & -1.451129 \\ \mathrm{H} & -2.429390 & -2.554585 & -1.643707 \\ \mathrm{H} & -1.234409 & 1.962853 & -0.748318 \\ \mathrm{H} & -0.596739 & 0.595763 & 1.914665\end{array}$

16

28

$\begin{array}{lrrr}\mathrm{P} & 0.885312 & -1.515142 & -0.746140 \\ \mathrm{O} & 4.285493 & 0.349428 & -0.121630 \\ \mathrm{O} & 2.351530 & -0.515381 & -0.807992 \\ \mathrm{O} & 0.453096 & 1.476401 & -1.375682 \\ \mathrm{O} & -3.198539 & -0.894647 & 1.542747 \\ \mathrm{O} & -2.574256 & -0.492286 & -0.626005 \\ \mathrm{O} & -0.644285 & 1.869751 & 0.589430 \\ \mathrm{~N} & -0.990052 & -0.703041 & 0.981344 \\ \mathrm{~N} & -0.010531 & -0.254958 & 0.101604 \\ \mathrm{C} & 3.235973 & -0.185134 & 0.170391 \\ \mathrm{C} & 2.853327 & -0.499717 & 1.607249 \\ \mathrm{C} & -3.970202 & -0.526232 & -1.004546 \\ \mathrm{C} & -2.352022 & -0.700156 & 0.691928 \\ \mathrm{C} & -0.768682 & 3.258874 & 0.198434 \\ \mathrm{C} & -0.022728 & 1.093356 & -0.331264 \\ \mathrm{H} & 3.706795 & -0.284017 & 2.256816 \\ \mathrm{H} & 2.562808 & -1.553754 & 1.732033 \\ \mathrm{H} & 1.999749 & 0.119963 & 1.916041 \\ \mathrm{H} & 1.613595 & -2.502947 & -0.026489 \\ \mathrm{H} & 1.029059 & -1.587686 & -2.149680 \\ \mathrm{H} & 0.223859 & 3.711352 & 0.085232 \\ \mathrm{H} & -4.530732 & 0.248303 & -0.467022 \\ \mathrm{H} & -4.402294 & -1.508986 & -0.779602 \\ \mathrm{H} & -3.979794 & -0.335651 & -2.081524 \\ \mathrm{H} & -1.324424 & 3.735961 & 1.010775 \\ \mathrm{H} & -1.315021 & 3.340070 & -0.749137 \\ \mathrm{H} & -0.775429 & -0.636459 & 1.973621 \\ \mathrm{H} & -0.322089 & -2.305096 & -0.709682\end{array}$


26

$\begin{array}{llll}\mathrm{P} & 1.604892 & -1.115206 & -0.651002\end{array}$

$\begin{array}{llll}\text { O } & 2.621059 & -0.508609 & 0.499477\end{array}$

$\begin{array}{llll}\mathrm{O} & 1.113106 & 1.850531 & -0.952407\end{array}$

$\begin{array}{llll}\mathrm{O} & -2.764361 & -1.153375 & 1.470578\end{array}$

$\begin{array}{llll}\mathrm{O} & -2.109128 & -0.877430 & -0.707377\end{array}$

$\begin{array}{llll}\mathrm{O} & -0.706767 & 1.997437 & 0.433059\end{array}$

$\begin{array}{llll}\mathrm{N} & 0.302550 & -0.019001 & 0.080850\end{array}$

$\begin{array}{llll}\mathrm{N} & -0.608635 & -0.571435 & 0.969337\end{array}$

$\begin{array}{llll}\text { C } & 4.023611 & -0.818382 & 0.525115\end{array}$

$\begin{array}{llll}\text { C } & 0.298534 & 1.328678 & -0.201868\end{array}$

$\begin{array}{llll}\text { C } & -3.449675 & -1.231256 & -1.108750\end{array}$

$\begin{array}{llll}\text { C } & -1.919405 & -0.877904 & 0.636590\end{array}$

$\begin{array}{llll}\text { C } & -0.764359 & 3.405660 & 0.124012\end{array}$

$\begin{array}{llll}\mathrm{H} & 4.589032 & 0.056242 & 0.175580\end{array}$

$\begin{array}{llll}\mathrm{H} & 4.292757 & -1.044722 & 1.564513\end{array}$

$\mathrm{H} \quad 4.261319 \quad-1.685553 \quad-0.110781$

$\begin{array}{llll}\mathrm{H} & 2.659586 & -1.994838 & -1.136733\end{array}$

$\mathrm{H} \quad 1.375326 \quad-0.527347 \quad-1.919876$

H $\quad 0.694263 \quad-2.184248 \quad-0.423992$

$\begin{array}{lrrr}\mathrm{H} & 0.175956 & 3.900807 & 0.397822\end{array}$

$\mathrm{H} \quad-4.176614 \quad-0.523160 \quad-0.691405$

$\mathrm{H} \quad-3.699987 \quad-2.244466 \quad-0.769310$

H $\quad-3.445847 \quad-1.180227 \quad-2.201891$

$\begin{array}{llll}\mathrm{H} & -1.595060 & 3.797762 & 0.719054\end{array}$

$\mathrm{H} \quad-0.951305 \quad 3.558894 \quad-0.946639$

$\mathrm{H} \quad-0.473713 \quad-0.377203 \quad 1.960159$

18

26

$\begin{array}{llll}\mathrm{P} & -0.540761 & -1.918151 & -0.455206\end{array}$

$\begin{array}{llll}\text { O } & 2.486571 & 0.711121 & 0.196508\end{array}$

$\begin{array}{llll}\mathrm{O} & 2.461061 & -1.531202 & -0.204634\end{array}$

$\begin{array}{llll}\mathrm{O} & -1.813971 & 0.666002 & -1.429703\end{array}$

$\begin{array}{llll}\mathrm{O} & -1.658582 & 2.531700 & -0.205533\end{array}$

$\begin{array}{llll}\mathrm{O} & -1.407052 & -1.351253 & 0.827635\end{array}$ 


$\begin{array}{lrrr}\mathrm{N} & 0.490398 & -0.323443 & -0.246183 \\ \mathrm{~N} & -0.020760 & 0.939075 & 0.080052 \\ \mathrm{C} & 3.912930 & 0.582275 & 0.342472 \\ \mathrm{C} & 1.851343 & -0.466041 & -0.081599 \\ \mathrm{C} & -2.603831 & -2.033600 & 1.239594 \\ \mathrm{C} & -1.087327 & 1.336015 & -0.492016 \\ \mathrm{C} & -0.961625 & 3.342380 & 0.763985 \\ \mathrm{H} & 4.370030 & 0.204640 & -0.581813 \\ \mathrm{H} & 4.270698 & 1.594160 & 0.560938 \\ \mathrm{H} & 4.164820 & -0.099342 & 1.165603 \\ \mathrm{H} & 0.691380 & -2.604666 & -0.373446 \\ \mathrm{H} & 0.050005 & 3.575232 & 0.408549 \\ \mathrm{H} & -3.400762 & -1.931603 & 0.488291 \\ \mathrm{H} & -2.915397 & -1.545058 & 2.170445 \\ \mathrm{H} & -2.577100 & 1.227230 & -1.662655 \\ \mathrm{H} & -2.420978 & -3.100606 & 1.430730 \\ \mathrm{H} & -1.562572 & 4.252056 & 0.862251 \\ \mathrm{H} & -1.370368 & -3.117393 & -0.505588 \\ \mathrm{H} & -0.890047 & 2.810188 & 1.721166 \\ \mathrm{H} & -0.798906 & -1.579103 & -1.802279\end{array}$

19

$\begin{array}{lrrr}26 & & & \\ & & & \\ \mathrm{P} & 0.604724 & -1.548581 & 0.768424 \\ \mathrm{O} & 1.621829 & -1.813187 & -0.558185 \\ \mathrm{O} & 1.596497 & 2.229974 & 0.419383 \\ \mathrm{O} & 1.333560 & 0.130133 & 1.012007 \\ \mathrm{O} & -2.619479 & -1.289949 & -1.016928 \\ \mathrm{O} & -2.381612 & 0.546865 & 0.321756 \\ \mathrm{~N} & 0.032565 & 1.021129 & -0.739270 \\ \mathrm{~N} & -0.555167 & -0.286666 & -0.767301 \\ \mathrm{C} & 3.005581 & -1.454401 & -0.514475 \\ \mathrm{C} & 1.211070 & 3.355708 & -0.399898 \\ \mathrm{C} & 0.939430 & 1.079834 & 0.204507 \\ \mathrm{C} & -3.783882 & 0.446695 & 0.667244 \\ \mathrm{C} & -1.955966 & -0.392977 & -0.535699 \\ \mathrm{H} & 3.512465 & -2.070454 & -1.269222 \\ \mathrm{H} & 3.446768 & -1.666477 & 0.473544 \\ \mathrm{H} & 3.162359 & -0.391100 & -0.749811\end{array}$




$\begin{array}{rrrr}\mathrm{H} & 1.832830 & 4.185278 & -0.048929 \\ \mathrm{H} & 1.440927 & -2.113695 & 1.765969 \\ \mathrm{H} & 1.404702 & 3.146240 & -1.459667 \\ \mathrm{H} & 0.145798 & 3.582280 & -0.266753 \\ \mathrm{H} & -4.404588 & 0.552521 & -0.230922 \\ \mathrm{H} & -3.991449 & -0.520389 & 1.141149 \\ \mathrm{H} & -3.966162 & 1.269365 & 1.364315 \\ \mathrm{H} & -0.420049 & -1.076498 & 1.651860 \\ \mathrm{H} & -0.330427 & -0.770810 & -1.641524 \\ \mathrm{H} & -0.149583 & -2.678624 & 0.335285\end{array}$

20

21

$\begin{array}{lrrr}\mathrm{P} & -0.589328 & -0.457970 & -0.134194 \\ \mathrm{O} & 3.383253 & 0.207427 & -0.745072 \\ \mathrm{O} & 1.151064 & 0.117414 & -0.720796 \\ \mathrm{O} & -2.140385 & -1.094153 & 0.339302 \\ \mathrm{O} & -1.125186 & 1.228182 & -0.243242 \\ \mathrm{C} & 2.382402 & -0.947375 & 1.100165 \\ \mathrm{C} & 2.327934 & -0.160722 & -0.213073 \\ \mathrm{C} & -3.299905 & -0.563715 & -0.252637 \\ \mathrm{C} & -0.594420 & 2.177664 & 0.639707 \\ \mathrm{H} & 3.391491 & -1.355374 & 1.231159 \\ \mathrm{H} & 2.162130 & -0.270102 & 1.938566 \\ \mathrm{H} & 1.634212 & -1.752316 & 1.123698 \\ \mathrm{H} & 0.243655 & 2.737816 & 0.180712 \\ \mathrm{H} & -4.137053 & -1.254861 & -0.047707 \\ \mathrm{H} & -3.557606 & 0.437691 & 0.143021 \\ \mathrm{H} & -3.199134 & -0.463837 & -1.353026 \\ \mathrm{H} & -1.381974 & 2.898046 & 0.931224 \\ \mathrm{H} & -0.893028 & -0.515311 & -1.534690 \\ \mathrm{H} & -0.212295 & 1.710618 & 1.573498 \\ \mathrm{H} & -0.204006 & -0.201442 & 1.234420 \\ \mathrm{H} & -0.052501 & -1.807446 & -0.094485\end{array}$


16

$\begin{array}{lrrr}\mathrm{P} & -0.891212 & 0.961230 & 0.233509 \\ \mathrm{O} & 1.195816 & -0.860703 & 1.106179 \\ \mathrm{O} & 0.731394 & 0.682859 & -0.483529 \\ \mathrm{O} & -1.325806 & -0.449337 & -0.478412 \\ \mathrm{C} & 2.901959 & -0.282366 & -0.514646 \\ \mathrm{C} & 1.535971 & -0.205781 & 0.131500 \\ \mathrm{C} & -2.601086 & -1.064979 & -0.245792 \\ \mathrm{H} & 3.533628 & -0.987571 & 0.033573 \\ \mathrm{H} & 3.369591 & 0.711432 & -0.528665 \\ \mathrm{H} & 2.799273 & -0.606632 & -1.559428 \\ \mathrm{H} & -3.321145 & -0.359243 & 0.197038 \\ \mathrm{H} & -2.980027 & -1.409953 & -1.215846 \\ \mathrm{H} & -2.466511 & -1.921776 & 0.428339 \\ \mathrm{H} & -2.238984 & 1.161460 & 0.738029 \\ \mathrm{H} & -0.867100 & 2.183382 & -0.479372 \\ \mathrm{H} & -0.292852 & 1.146638 & 1.503416\end{array}$

22

18

$\begin{array}{lrrr}\mathrm{P} & -0.429150 & 1.596540 & -0.000096 \\ \mathrm{O} & 1.172806 & 0.783419 & -0.000149 \\ \mathrm{O} & 0.873828 & -1.486623 & -0.000417 \\ \mathrm{O} & -1.139125 & 0.168010 & -1.073399 \\ \mathrm{O} & -1.138823 & 0.168048 & 1.073791 \\ \mathrm{C} & 3.103164 & -0.565895 & 0.000229 \\ \mathrm{C} & 1.589249 & -0.495020 & -0.000238 \\ \mathrm{C} & -2.158975 & -1.773366 & 0.000161 \\ \mathrm{C} & -1.432484 & -0.477510 & 0.000284 \\ \mathrm{H} & 3.502363 & -0.047791 & -0.882169 \\ \mathrm{H} & 3.501194 & -0.052717 & 0.886066 \\ \mathrm{H} & 3.424461 & -1.611811 & -0.002277 \\ \mathrm{H} & 0.030593 & 2.353223 & 1.102694 \\ \mathrm{H} & 0.030197 & 2.353066 & -1.103149 \\ \mathrm{H} & -3.240997 & -1.572117 & -0.011540 \\ \mathrm{H} & -1.913258 & -2.338336 & 0.904117\end{array}$




$\begin{array}{lrrr}\mathrm{H} & -1.896253 & -2.346787 & -0.893721 \\ \mathrm{H} & -1.756247 & 2.123085 & 0.000188\end{array}$

23

18

$\begin{array}{llll}\mathrm{P} & 1.309925 & -1.303167 & 0.003867\end{array}$

$\begin{array}{llll}\mathrm{O} & 1.006320 & 0.097049 & -1.073907\end{array}$

$\begin{array}{lrrr}\mathrm{O} & 0.991010 & 0.096931 & 1.078403\end{array}$

$\begin{array}{llll}\mathrm{O} & -1.048275 & -1.291576 & -0.011039\end{array}$

$\begin{array}{llll}\mathrm{O} & -0.931541 & 0.982974 & -0.011839\end{array}$

$\begin{array}{llll}\text { C } & 1.084258 & 2.311026 & 0.003102\end{array}$

$\begin{array}{llll}\text { C } & 0.614313 & 0.877368 & -0.000428\end{array}$

$\begin{array}{lrrr}\text { C } & -3.088668 & -0.002996 & 0.006787\end{array}$

$\begin{array}{llll}\text { C } & -1.590154 & -0.175017 & -0.010612\end{array}$

$\mathrm{H} \quad 2.728072 \quad-1.297659 \quad 0.013773$

$\begin{array}{llll}\mathrm{H} & 2.182980 & 2.316611 & 0.010491\end{array}$

$\mathrm{H} \quad 1.067193 \quad-2.209122 \quad-1.068082$

$\begin{array}{llll}\mathrm{H} & 1.052157 & -2.209530 & 1.071975\end{array}$

H $\quad 0.721869 \quad 2.827996 \quad-0.895854$

$\begin{array}{lrrr}\mathrm{H} & 0.709828 & 2.827503 & 0.897402\end{array}$

$\mathrm{H} \quad-3.573931 \quad-0.971785 \quad-0.161651$

$\begin{array}{llll}\mathrm{H} & -3.399437 & 0.722606 & -0.758926\end{array}$

$\begin{array}{llll}\mathrm{H} & -3.396211 & 0.395575 & 0.986818\end{array}$

2d

16

$\begin{array}{llll}\text { O } & 2.439194 & -0.690225 & -0.118343\end{array}$

$\begin{array}{llll}\text { O } & 2.138241 & 1.571604 & 0.060037\end{array}$

$\begin{array}{llll}\text { O } & -2.439210 & 0.690219 & 0.118400\end{array}$

$\begin{array}{llll}\mathrm{O} & -2.138215 & -1.571593 & -0.060128\end{array}$

$\begin{array}{llll}\mathrm{N} & 0.356233 & 0.187436 & -0.477063\end{array}$

$\begin{array}{llll}\mathrm{N} & -0.356229 & -0.187422 & 0.477037\end{array}$

$\begin{array}{llll}\text { C } & 3.868106 & -0.523398 & 0.089271\end{array}$

$\begin{array}{llll}\text { C } & 1.734345 & 0.445087 & -0.107644\end{array}$

$\begin{array}{llll}\text { C } & -3.868122 & 0.523377 & -0.089204\end{array}$

$\begin{array}{llll}\text { C } & -1.734339 & -0.445078 & 0.107618\end{array}$ 


$\begin{array}{rrrr}\mathrm{H} & 4.289802 & -1.526969 & -0.009853 \\ \mathrm{H} & 4.281315 & 0.153967 & -0.667653 \\ \mathrm{H} & 4.056383 & -0.117218 & 1.090068 \\ \mathrm{H} & -4.289838 & 1.526931 & 0.010009 \\ \mathrm{H} & -4.281303 & -0.154061 & 0.667669 \\ \mathrm{H} & -4.056405 & 0.117275 & -1.090033\end{array}$

3d

20

$\begin{array}{llll}\mathrm{P} & -0.022484 & 1.886066 & -0.340111\end{array}$

$\begin{array}{llll}\mathrm{O} & 2.127838 & 1.030333 & 0.507095\end{array}$

$\begin{array}{llll}\text { O } & 2.028219 & -1.198858 & -0.059597\end{array}$

$\begin{array}{llll}\mathrm{O} & -2.524438 & -1.653849 & -0.692120\end{array}$

$\begin{array}{llll}\mathrm{O} & -1.734393 & 0.052462 & 0.645569\end{array}$

$\begin{array}{llll}\mathrm{N} & 0.359476 & 0.262631 & -0.633985\end{array}$

$\mathrm{N} \quad-0.508016 \quad-0.669919 \quad-1.212825$

$\begin{array}{llll}\text { C } & 3.312523 & -1.405562 & 0.583240\end{array}$

$\begin{array}{llll}\text { C } & 1.572592 & 0.043238 & -0.012885\end{array}$

$\begin{array}{llll}\text { C } & -2.963811 & -0.077310 & 1.377302\end{array}$

$\begin{array}{llll}\text { C } & -1.627284 & -0.857045 & -0.468758\end{array}$

$\begin{array}{llll}\mathrm{H} & 4.086253 & -0.809184 & 0.085112\end{array}$

$\begin{array}{llll}\mathrm{H} & 3.513520 & -2.474079 & 0.470408\end{array}$

$\mathrm{H} \quad 3.255567 \quad-1.129045 \quad 1.642824$

$\begin{array}{llll}\mathrm{H} & 0.859442 & 2.828274 & -0.919344\end{array}$

$\begin{array}{llll}\mathrm{H} & -3.830641 & 0.143852 & 0.739019\end{array}$

$\begin{array}{llll}\mathrm{H} & -3.073578 & -1.096732 & 1.769524\end{array}$

$\begin{array}{llll}\mathrm{H} & -2.904541 & 0.641138 & 2.204128\end{array}$

$\mathrm{H} \quad-1.238243 \quad 2.060475 \quad-1.024454$

$\mathrm{H} \quad-0.232662 \quad 2.334713 \quad 0.981138$

\section{4}

21

$\begin{array}{llll}\mathrm{P} & -0.433430 & 2.018148 & -0.073529\end{array}$

$\begin{array}{llll}\text { O } & 2.682360 & -0.947015 & -0.416976\end{array}$

$\begin{array}{llll}\mathrm{O} & 1.544997 & 0.507160 & 0.946598\end{array}$

$\begin{array}{llll}\text { O } & -2.406119 & 0.687335 & 0.521255\end{array}$

$\begin{array}{llll}\mathrm{O} & -1.864112 & -1.418034 & -0.230645\end{array}$ 


$\begin{array}{lrrr}\mathrm{N} & 0.590659 & -0.334879 & -0.949448 \\ \mathrm{~N} & -0.535597 & 0.412823 & -0.661361 \\ \mathrm{C} & 3.864966 & -0.881799 & 0.444736 \\ \mathrm{C} & 1.648279 & -0.215851 & -0.038905 \\ \mathrm{C} & -3.080119 & -1.995892 & 0.366542 \\ \mathrm{C} & -1.698387 & -0.136557 & -0.055790 \\ \mathrm{H} & 4.597547 & -1.528322 & -0.042692 \\ \mathrm{H} & 4.225471 & 0.150915 & 0.501567 \\ \mathrm{H} & 3.612016 & -1.254614 & 1.442757 \\ \mathrm{H} & 0.777230 & 2.497916 & -0.604883 \\ \mathrm{H} & 0.763677 & -0.553651 & -1.929472 \\ \mathrm{H} & -3.959955 & -1.553425 & -0.111473 \\ \mathrm{H} & -3.082885 & -1.800715 & 1.443761 \\ \mathrm{H} & -3.006892 & -3.064087 & 0.154615 \\ \mathrm{H} & -1.442040 & 2.811800 & -0.652485 \\ \mathrm{H} & -0.433594 & 2.221372 & 1.315540\end{array}$

5

14

$\begin{array}{llll}\mathrm{P} & 0.000079 & -0.000061 & -0.116359\end{array}$

$\begin{array}{llll}\text { O } & 1.601123 & 0.608245 & -0.228213\end{array}$

$\begin{array}{llll}\text { O } & -1.601219 & -0.608151 & -0.228170\end{array}$

$\begin{array}{llll}\text { C } & 2.619243 & -0.246172 & 0.262623\end{array}$

$\begin{array}{llll}\text { C } & -2.619293 & 0.246220 & 0.262626\end{array}$

$\begin{array}{lllr}\mathrm{H} & 3.592408 & 0.199863 & 0.019641\end{array}$

$\mathrm{H} \quad 2.568098 \quad-1.250191 \quad-0.204720$

$\mathrm{H} \quad 2.552401 \quad-0.374129 \quad 1.360121$

$\begin{array}{llll}\mathrm{H} & 0.383486 & -1.232790 & -0.707259\end{array}$

$\mathrm{H} \quad 0.000566 \quad-0.001188 \quad 1.308121$

$\begin{array}{llll}\mathrm{H} & -3.592574 & -0.196921 & 0.014659\end{array}$

$\mathrm{H} \quad-2.564840 \quad 1.252082 \quad-0.200629$

$\begin{array}{lllr}\mathrm{H} & -2.555843 & 0.369913 & 1.360845\end{array}$

$\begin{array}{llll}\mathrm{H} & -0.383817 & 1.233238 & -0.705832\end{array}$

6

18

$\begin{array}{llll}\text { O } & 2.625166 & 0.172616 & -0.490125\end{array}$ 


$\begin{array}{lrrr}\mathrm{O} & 1.241153 & -0.559819 & 1.187086 \\ \mathrm{O} & -2.625206 & 0.172703 & 0.490078 \\ \mathrm{O} & -1.241132 & -0.559935 & -1.186982 \\ \mathrm{~N} & 0.665174 & 1.209964 & -0.190274 \\ \mathrm{~N} & -0.665166 & 1.209937 & 0.190203 \\ \mathrm{C} & 3.601905 & -0.809101 & -0.079840 \\ \mathrm{C} & 1.486581 & 0.173726 & 0.251361 \\ \mathrm{C} & -3.601901 & -0.809128 & 0.079866 \\ \mathrm{C} & -1.486574 & 0.173725 & -0.251378 \\ \mathrm{H} & 4.447440 & -0.677504 & -0.761550 \\ \mathrm{H} & 3.911162 & -0.633218 & 0.958244 \\ \mathrm{H} & 3.187734 & -1.821158 & -0.168467 \\ \mathrm{H} & 0.843395 & 1.572267 & -1.123663 \\ \mathrm{H} & -4.445691 & -0.680377 & 0.764277 \\ \mathrm{H} & -3.914315 & -0.630697 & -0.956851 \\ \mathrm{H} & -3.186264 & -1.820906 & 0.164490 \\ \mathrm{H} & -0.843420 & 1.572428 & 1.123503\end{array}$

7

9

$\begin{array}{llll}\mathrm{P} & -0.749284 & -0.668949 & 0.000000\end{array}$

$\begin{array}{llll}\mathrm{O} & 0.000000 & 0.702213 & 0.000000\end{array}$

$\begin{array}{llll}\text { C } & 1.479421 & 0.838736 & 0.000000\end{array}$

$\begin{array}{lllr}\mathrm{H} & 1.884637 & 0.379254 & 0.908602\end{array}$

H $\quad 1.884637 \quad 0.379254 \quad-0.908602$

$\mathrm{H} \quad 1.649391 \quad 1.917564 \quad 0.000000$

$\begin{array}{llll}\mathrm{H} & -2.120799 & -0.357394 & 0.000000\end{array}$

$\begin{array}{llll}\mathrm{H} & -0.467571 & -1.467278 & 1.133410\end{array}$

$\mathrm{H} \quad-0.467571 \quad-1.467278 \quad-1.133410$

8

23

$\begin{array}{llll}\text { O } & 2.366651 & -0.764852 & 0.226952\end{array}$

$\begin{array}{llll}\mathrm{O} & 0.617056 & -1.878758 & -0.701658\end{array}$

$\begin{array}{llll}\mathrm{O} & 0.183818 & 2.672202 & -0.282355\end{array}$

$\begin{array}{llll}\mathrm{O} & -2.961915 & -0.269774 & -0.506489\end{array}$

$\begin{array}{llll}\text { O } & -1.600233 & -0.131979 & 1.336655\end{array}$ 


$\begin{array}{lrrr}\mathrm{N} & 0.483513 & 0.407709 & -0.392815 \\ \mathrm{~N} & -0.836137 & 0.330124 & -0.794112 \\ \mathrm{C} & 3.064376 & -2.032294 & 0.315653 \\ \mathrm{C} & 2.344915 & 1.941213 & 0.431544 \\ \mathrm{C} & 1.133645 & -0.853659 & -0.310324 \\ \mathrm{C} & 0.943806 & 1.746354 & -0.092314 \\ \mathrm{C} & -4.058702 & -0.621441 & 0.363919 \\ \mathrm{C} & -1.786499 & -0.047449 & 0.139711 \\ \mathrm{H} & 4.033305 & -1.792556 & 0.763175 \\ \mathrm{H} & 3.192092 & -2.468566 & -0.682303 \\ \mathrm{H} & 3.096716 & 1.544445 & -0.261253 \\ \mathrm{H} & 2.503392 & -2.729128 & 0.949408 \\ \mathrm{H} & 2.485086 & 3.018553 & 0.561430 \\ \mathrm{H} & 2.483846 & 1.425354 & 1.389241 \\ \mathrm{H} & -4.918317 & -0.762257 & -0.298149 \\ \mathrm{H} & -4.252663 & 0.184977 & 1.082286 \\ \mathrm{H} & -3.834969 & -1.547094 & 0.908985 \\ \mathrm{H} & -1.012388 & 0.150386 & -1.778300\end{array}$

9

11

$\begin{array}{llll}\text { O } & 1.185616 & 0.762178 & 0.000000\end{array}$

$\begin{array}{llll}\mathrm{O} & -0.484532 & -0.775792 & 0.000000\end{array}$

$\begin{array}{llll}\text { C } & 0.523358 & -1.811892 & 0.000000\end{array}$

$\begin{array}{llll}\text { C } & 0.000000 & 0.498146 & 0.000000\end{array}$

$\begin{array}{llll}\text { C } & -1.130371 & 1.499256 & 0.000000\end{array}$

$\mathrm{H} \quad 1.156149 \quad-1.732356 \quad 0.893126$

$\mathrm{H} \quad 1.156149 \quad-1.732356 \quad-0.893126$

$\begin{array}{llll}\mathrm{H} & -1.765183 & 1.352080 & 0.884057\end{array}$

$\begin{array}{llll}\mathrm{H} & -1.765183 & 1.352080 & -0.884057\end{array}$

$\begin{array}{llll}\mathrm{H} & -0.719288 & 2.512634 & 0.000000\end{array}$

$\begin{array}{llll}\mathrm{H} & -0.029238 & -2.756227 & 0.000000\end{array}$

\section{$\mathrm{AcO}^{-}$}

7

$\begin{array}{llll}\text { O } & 1.118447 & 0.800302 & 0.000000\end{array}$ 


$\begin{array}{rrrr}\mathrm{O} & -1.161072 & 0.711696 & 0.000000 \\ \mathrm{C} & 0.041859 & -1.357648 & 0.000000 \\ \mathrm{C} & 0.000000 & 0.212117 & 0.000000 \\ \mathrm{H} & 1.073227 & -1.740314 & 0.000000 \\ \mathrm{H} & -0.491691 & -1.741244 & 0.885243 \\ \mathrm{H} & -0.491691 & -1.741244 & -0.885243\end{array}$

$\mathrm{AcOH}$

8

$\begin{array}{llll}\text { O } & 0.166251 & 1.357933 & 0.000000\end{array}$

$\begin{array}{llll}\mathrm{O} & -1.245386 & -0.411424 & 0.000000\end{array}$

$\begin{array}{llll}\text { C } & 1.081800 & -0.893092 & 0.000000\end{array}$

$\begin{array}{llll}\text { C } & 0.000000 & 0.155917 & 0.000000\end{array}$

$\begin{array}{llll}\mathrm{H} & 2.062141 & -0.409648 & 0.000000\end{array}$

$\begin{array}{llll}\mathrm{H} & 0.978994 & -1.537396 & 0.883711\end{array}$

$\mathrm{H} \quad 0.978994 \quad-1.537396 \quad-0.883711$

$\begin{array}{llll}\mathrm{H} & -1.877848 & 0.335412 & 0.000000\end{array}$

$E_{15-7}$

27

$\begin{array}{llll}\mathrm{P} & 0.421893 & -1.141446 & 1.140306\end{array}$

$\begin{array}{llll}\text { O } & 1.868565 & -1.940812 & -0.612414\end{array}$

$\begin{array}{llll}\text { O } & 1.563607 & 2.357893 & 0.335493\end{array}$

$\begin{array}{llll}\text { O } & 1.388100 & 0.252220 & 0.967904\end{array}$

$\begin{array}{llll}\text { O } & -2.601314 & 0.456630 & 0.078792\end{array}$

$\begin{array}{llll}\mathrm{O} & -2.358841 & -1.637151 & -0.822486\end{array}$

$\begin{array}{llll}\mathrm{N} & -0.507034 & -0.285155 & -0.530435\end{array}$

$\begin{array}{llll}\mathrm{N} & -0.091332 & 1.076777 & -0.639985\end{array}$

$\begin{array}{llll}\text { C } & 3.295712 & -1.656065 & -0.726917\end{array}$

$\begin{array}{llll}\text { C } & 1.125660 & 3.496446 & -0.475432\end{array}$

$\begin{array}{llll}\text { C } & 0.904103 & 1.239208 & 0.182361\end{array}$

$\begin{array}{llll}\text { C } & -4.051908 & 0.273805 & 0.208540\end{array}$

$\begin{array}{llll}\text { C } & -1.958116 & -0.557006 & -0.462388\end{array}$

$\begin{array}{llll}\mathrm{H} & 3.859697 & -2.168476 & 0.062998\end{array}$

$\mathrm{H} \quad 3.652222 \quad-1.969024 \quad-1.716115$

H $\quad 3.408970 \quad-0.574464 \quad-0.618479$ 


$\begin{array}{lrrr}\mathrm{H} & 1.799300 & 4.307364 & -0.191274 \\ \mathrm{H} & 1.744476 & -2.901219 & -0.715617 \\ \mathrm{H} & 1.378084 & -1.681374 & 2.027117 \\ \mathrm{H} & 1.223933 & 3.250829 & -1.538585 \\ \mathrm{H} & 0.085334 & 3.740491 & -0.234450 \\ \mathrm{H} & -4.488245 & 0.130504 & -0.785587 \\ \mathrm{H} & -4.407732 & 1.197983 & 0.668137 \\ \mathrm{H} & -4.257887 & -0.594644 & 0.843254 \\ \mathrm{H} & -0.586926 & -0.674421 & 2.012991 \\ \mathrm{H} & -0.176122 & -2.340046 & 0.666934 \\ \mathrm{H} & -0.148579 & -0.831730 & -1.328274\end{array}$

$\mathrm{H}_{3} \mathrm{P}=\mathrm{O}$

5

$\begin{array}{rrrr}\mathrm{P} & -0.384012 & 0.000002 & -0.000007 \\ \mathrm{O} & 1.107296 & 0.000001 & 0.000006 \\ \mathrm{H} & -1.032746 & 1.168443 & -0.500194 \\ \mathrm{H} & -1.032743 & -0.151025 & 1.261993 \\ \mathrm{H} & -1.032702 & -1.017462 & -0.761739\end{array}$

$\mathrm{MeO}^{-}$

5

$\begin{array}{llll}\text { O } & -0.000000 & -0.000000 & 0.794791\end{array}$

$\begin{array}{llll}\text { C } & 0.000000 & 0.000000 & -0.536911\end{array}$

$\mathrm{H} \quad 0.895770 \quad-0.517173 \quad-1.045622$

$\begin{array}{llll}\mathrm{H} & 0.000000 & 1.034346 & -1.045622\end{array}$

$\mathrm{H} \quad-0.895770 \quad-0.517173 \quad-1.045622$

$\mathrm{MeOH}$

6

$\begin{array}{llll}\mathrm{O} & -0.046927 & -0.761946 & 0.000000\end{array}$

$\begin{array}{llll}\text { C } & -0.046927 & 0.667844 & 0.000000\end{array}$

$\begin{array}{llll}\mathrm{H} & 0.874639 & -1.064203 & 0.000000\end{array}$ 


$\begin{array}{rrrr}\mathrm{H} & 0.440094 & 1.085429 & 0.898102 \\ \mathrm{H} & 0.440094 & 1.085429 & -0.898102 \\ \mathrm{H} & -1.097846 & 0.981848 & 0.000000\end{array}$

\section{$\mathrm{PH}_{3}$}

4

$\begin{array}{lrrr}\mathrm{P} & -0.000000 & -0.000078 & -0.131470 \\ \mathrm{H} & 1.032621 & -0.596285 & 0.657607 \\ \mathrm{H} & 0.000430 & 1.192993 & 0.656834 \\ \mathrm{H} & -1.033051 & -0.595540 & 0.657607\end{array}$

$T_{15-7}$

27

$\begin{array}{lrrr}\mathrm{P} & 0.900153 & -1.557652 & 0.644939 \\ \mathrm{O} & 2.075044 & -0.878840 & -0.620049 \\ \mathrm{O} & 0.668172 & 0.288130 & 1.275581 \\ \mathrm{O} & 0.613676 & 2.379773 & 0.560311 \\ \mathrm{O} & -2.344618 & -1.838608 & -0.761035 \\ \mathrm{O} & -2.223577 & 0.263641 & 0.144402 \\ \mathrm{~N} & 0.227206 & 0.651156 & -0.964988 \\ \mathrm{~N} & -0.344481 & -0.677703 & -0.806331 \\ \mathrm{C} & 3.447702 & -0.614555 & -0.212880 \\ \mathrm{C} & 0.463147 & 1.110796 & 0.324176 \\ \mathrm{C} & 0.319115 & 3.359520 & -0.496229 \\ \mathrm{C} & -3.631395 & 0.206532 & 0.566217 \\ \mathrm{C} & -1.785251 & -0.811860 & -0.481074 \\ \mathrm{H} & 3.952255 & -0.164268 & -1.074957 \\ \mathrm{H} & 3.932866 & -1.565826 & 0.033723 \\ \mathrm{H} & 3.480751 & 0.067964 & 0.647244 \\ \mathrm{H} & 1.931373 & -1.707741 & 1.597968 \\ \mathrm{H} & 1.399291 & 0.102672 & -1.040522 \\ \mathrm{H} & 1.166797 & 3.404357 & -1.187756 \\ \mathrm{H} & 0.930177 & -2.792317 & -0.047240 \\ \mathrm{H} & 0.205042 & 4.305111 & 0.038031 \\ \mathrm{H} & -4.267778 & 0.104990 & -0.319002 \\ \mathrm{H} & -3.807430 & 1.154689 & 1.077838\end{array}$




$\begin{array}{rrrr}\mathrm{H} & -3.773357 & -0.644525 & 1.240198 \\ \mathrm{H} & -0.597025 & 3.076675 & -1.022636 \\ \mathrm{H} & -0.225176 & -1.814030 & 1.471341 \\ \mathrm{H} & -0.198639 & -1.192509 & -1.684022\end{array}$

$T_{16-8}$

28

$\begin{array}{llll}\mathrm{P} & 1.197963 & -1.425442 & -1.441529\end{array}$

$\begin{array}{lllr}\text { O } & 2.874262 & 0.755684 & 0.503239\end{array}$

$\begin{array}{llll}\text { O } & 2.297108 & -1.470701 & -0.341442\end{array}$

$\begin{array}{llll}\text { O } & 0.823818 & 1.779209 & -1.346801\end{array}$

$\begin{array}{llll}\mathrm{O} & -2.849551 & -0.754884 & 1.620745\end{array}$

$\begin{array}{llll}\mathrm{O} & -2.080306 & -0.977660 & -0.524500\end{array}$

$\begin{array}{llll}\mathrm{O} & -0.770276 & 2.189980 & 0.239461\end{array}$

$\begin{array}{lrrr}\mathrm{N} & 0.287043 & 0.136225 & 0.155882\end{array}$

$\begin{array}{llll}\mathrm{N} & -0.678947 & -0.231240 & 1.113121\end{array}$

$\begin{array}{llll}\text { C } & 2.179032 & -0.167526 & 0.782333\end{array}$

$\begin{array}{llll}\text { C } & 2.006751 & -0.878004 & 2.091026\end{array}$

$\begin{array}{llll}\text { C } & 0.155059 & 1.406146 & -0.390948\end{array}$

$\begin{array}{llll}\text { C } & -3.394063 & -1.461277 & -0.884037\end{array}$

$\begin{array}{llll}\text { C } & -1.957659 & -0.651617 & 0.796136\end{array}$

$\begin{array}{llll}\text { C } & -0.919574 & 3.508308 & -0.329680\end{array}$

$\mathrm{H} \quad 2.944160 \quad-1.416873 \quad 2.284674$

$\mathrm{H} \quad 1.861261 \quad-0.134563 \quad 2.885444$

H $\quad 1.736237 \quad-2.325884 \quad-2.405365$

H $\quad 1.177788 \quad-1.589205 \quad 2.079977$

$\mathrm{H} \quad 1.030555 \quad-0.229120 \quad-2.173544$

$\mathrm{H} \quad 0.027273 \quad 4.060075 \quad-0.275249$

$\mathrm{H} \quad-4.155867 \quad-0.704283 \quad-0.661200$

H $\quad-3.631575 \quad-2.381596 \quad-0.335602$

$\mathrm{H} \quad-3.346790 \quad-1.653808 \quad-1.960490$

$\begin{array}{llll}\mathrm{H} & -1.689673 & 3.998993 & 0.273764\end{array}$

$\mathrm{H} \quad-1.235415 \quad 3.441588 \quad-1.378722$

$\begin{array}{llll}\mathrm{H} & -0.661824 & 0.278160 & 1.996455\end{array}$

H $\quad-0.059959 \quad-2.005962 \quad-1.184816$ 


\section{$T_{17-5}$}

32

$\begin{array}{lrrr}\mathrm{P} & -1.631746 & -0.924483 & -0.319865 \\ \mathrm{O} & 2.861985 & -0.172501 & -2.002346 \\ \mathrm{O} & 2.123276 & -0.887932 & 0.046760 \\ \mathrm{O} & 0.793142 & 2.404131 & 0.226760 \\ \mathrm{O} & -2.601950 & -0.024606 & -1.301112 \\ \mathrm{O} & -1.011048 & 1.691340 & 1.439093 \\ \mathrm{O} & -0.187586 & -2.236350 & 0.997449 \\ \mathrm{~N} & 0.737074 & 0.320294 & -1.300671 \\ \mathrm{~N} & -0.208492 & 0.409049 & -0.288986 \\ \mathrm{C} & 3.425148 & -1.495966 & 0.225089 \\ \mathrm{C} & 1.986033 & -0.227760 & -1.159608 \\ \mathrm{C} & 0.824190 & 3.567971 & 1.077123 \\ \mathrm{C} & -4.007730 & -0.025344 & -1.021856 \\ \mathrm{C} & -0.409217 & -2.126329 & 2.414587 \\ \mathrm{C} & -0.206808 & 1.514468 & 0.530557 \\ \mathrm{H} & 4.210132 & -0.733060 & 0.167716 \\ \mathrm{H} & 3.602701 & -2.258515 & -0.542921 \\ \mathrm{H} & 3.400411 & -1.948393 & 1.221448 \\ \mathrm{H} & 1.657768 & 4.173696 & 0.707186 \\ \mathrm{H} & 0.991508 & 3.281442 & 2.123582 \\ \mathrm{H} & 0.642714 & -1.754349 & 0.768801 \\ \mathrm{H} & 0.621266 & 0.897751 & -2.131528 \\ \mathrm{H} & 0.394245 & -2.633685 & 2.969196 \\ \mathrm{H} & -4.480192 & 0.622745 & -1.769576 \\ \mathrm{H} & -4.430857 & -1.039820 & -1.100773 \\ \mathrm{H} & -4.212202 & 0.379617 & -0.017255 \\ \mathrm{H} & -2.496085 & -2.088395 & -0.227366 \\ \mathrm{H} & -1.921280 & -0.462001 & 0.988704 \\ \mathrm{H} & -1.359297 & -2.628836 & 2.630339 \\ \mathrm{H} & -0.743843 & -1.532451 & -1.250556 \\ \mathrm{H} & -0.474517 & -1.074446 & 2.732675 \\ \mathrm{H} & -0.118613 & 4.125653 & 1.007722\end{array}$




\section{$T_{\text {21-9Ret }}$}

16

$\begin{array}{lrrr}\mathrm{P} & 1.618988 & -0.349065 & 0.077896 \\ \mathrm{O} & 0.421555 & -1.334026 & 0.252754 \\ \mathrm{O} & 0.102500 & 0.846085 & -0.539349 \\ \mathrm{O} & -1.563826 & -0.852187 & -1.184203 \\ \mathrm{C} & -1.678827 & -0.165826 & 1.143781 \\ \mathrm{C} & -1.045445 & -0.443043 & -0.192129 \\ \mathrm{C} & -0.072055 & 2.155425 & -0.024067 \\ \mathrm{H} & 2.660152 & -1.231539 & 0.512897 \\ \mathrm{H} & 2.176071 & -0.003007 & -1.175074 \\ \mathrm{H} & 1.900305 & 0.706149 & 0.987098 \\ \mathrm{H} & 0.673236 & 2.823393 & -0.485362 \\ \mathrm{H} & 0.046569 & 2.204048 & 1.074892 \\ \mathrm{H} & -2.516600 & 0.530845 & 1.006406 \\ \mathrm{H} & -2.078384 & -1.116527 & 1.519207 \\ \mathrm{H} & -1.073340 & 2.528349 & -0.287982 \\ \mathrm{H} & -0.976694 & 0.235941 & 1.880353\end{array}$

$\mathrm{T}_{22-23}$

18

$\begin{array}{lrrr}\mathrm{P} & 0.982600 & -1.452528 & 0.005788 \\ \mathrm{O} & 1.048375 & -0.004342 & -1.065293 \\ \mathrm{O} & 1.021437 & -0.001165 & 1.074716 \\ \mathrm{O} & -1.071344 & -1.113905 & -0.019455 \\ \mathrm{O} & -0.852334 & 1.139226 & -0.021140 \\ \mathrm{C} & 1.415034 & 2.182963 & 0.006017 \\ \mathrm{C} & 0.854913 & 0.793998 & 0.001190 \\ \mathrm{C} & -3.051176 & 0.225655 & 0.010992 \\ \mathrm{C} & -1.553212 & 0.058091 & -0.019765 \\ \mathrm{H} & 2.510356 & 2.111762 & 0.020084 \\ \mathrm{H} & 2.385724 & -1.659347 & 0.023418 \\ \mathrm{H} & 1.097688 & 2.719147 & -0.894413 \\ \mathrm{H} & 1.074821 & 2.722391 & 0.896083 \\ \mathrm{H} & 0.657766 & -2.311305 & -1.072947 \\ \mathrm{H} & 0.631246 & -2.307961 & 1.078926 \\ \mathrm{H} & -3.534957 & -0.618962 & -0.491446\end{array}$




$\begin{array}{rrrr}\mathrm{H} & -3.378985 & 0.234135 & 1.061008 \\ \mathrm{H} & -3.345087 & 1.175299 & -0.448772\end{array}$

\section{$T_{23-12}$}

18

$\begin{array}{llll}\mathrm{P} & 1.808970 & -1.074721 & -0.041836\end{array}$

$\begin{array}{llll}\text { O } & 1.138271 & -0.049833 & -1.011896\end{array}$

$\begin{array}{llll}\mathrm{O} & 0.775705 & 0.403656 & 1.278265\end{array}$

$\begin{array}{llll}\mathrm{O} & -1.154694 & -1.492855 & 0.086205\end{array}$

$\begin{array}{llll}\mathrm{O} & -1.063184 & 0.786677 & -0.126987\end{array}$

$\begin{array}{llll}\text { C } & 0.733226 & 2.340893 & -0.199437\end{array}$

$\begin{array}{llll}\text { C } & 0.354017 & 0.934054 & 0.207713\end{array}$

$\begin{array}{llll}\text { C } & -3.198638 & -0.212875 & -0.080544\end{array}$

$\begin{array}{llll}\text { C } & -1.702398 & -0.413613 & -0.021633\end{array}$

$\begin{array}{llll}\mathrm{H} & 2.916423 & -0.648740 & 0.718128\end{array}$

$\begin{array}{llll}\mathrm{H} & 2.427046 & -1.945585 & -0.975779\end{array}$

$\begin{array}{llll}\mathrm{H} & 1.818047 & 2.459167 & -0.106272\end{array}$

H $\quad 1.057801 \quad-1.962301 \quad 0.743536$

$\mathrm{H} \quad 0.417576 \quad 2.561956 \quad-1.224462$

$\begin{array}{llll}\mathrm{H} & 0.240621 & 3.041529 & 0.488938\end{array}$

$\begin{array}{llll}\mathrm{H} & -3.696722 & -1.183827 & -0.156467\end{array}$

$\begin{array}{llll}\mathrm{H} & -3.532525 & 0.302615 & 0.831221\end{array}$

$\begin{array}{llll}\mathrm{H} & -3.468840 & 0.424098 & -0.932605\end{array}$

\section{$\mathrm{T}_{4-7}$}

27

$\begin{array}{lrrr}\mathrm{P} & 0.394939 & 1.270803 & 1.102469 \\ \mathrm{O} & 1.997292 & -2.188285 & -0.237500 \\ \mathrm{O} & 1.856082 & 0.045277 & 0.256133 \\ \mathrm{O} & 0.412854 & 2.139557 & -0.497885 \\ \mathrm{O} & -2.739673 & 1.021250 & 0.099791 \\ \mathrm{O} & -2.509372 & -1.240000 & -0.195641 \\ \mathrm{~N} & 0.032473 & -1.105502 & -0.405608 \\ \mathrm{~N} & -0.660811 & 0.112005 & -0.258438 \\ \mathrm{C} & 3.425054 & -2.185994 & 0.098346 \\ \mathrm{C} & 1.597282 & 2.729092 & -1.095597\end{array}$




$\begin{array}{lrrr}\mathrm{C} & 1.344471 & -1.056382 & -0.104660 \\ \mathrm{C} & -3.959935 & -1.416346 & -0.084981 \\ \mathrm{C} & -2.081939 & 0.026709 & -0.090626 \\ \mathrm{H} & 3.958525 & -1.497240 & -0.565031 \\ \mathrm{H} & 3.743784 & -3.216487 & -0.070120 \\ \mathrm{H} & 3.558177 & -1.899689 & 1.146835 \\ \mathrm{H} & 2.277574 & 1.951800 & -1.461829 \\ \mathrm{H} & 2.095397 & 3.350109 & -0.341458 \\ \mathrm{H} & 1.468269 & 2.029938 & 1.618846 \\ \mathrm{H} & 1.247415 & 3.366115 & -1.914905 \\ \mathrm{H} & 0.349979 & 0.220003 & 2.063088 \\ \mathrm{H} & -4.456823 & -0.854352 & -0.882731 \\ \mathrm{H} & -4.297190 & -1.062437 & 0.894836 \\ \mathrm{H} & -4.122262 & -2.490643 & -0.196333 \\ \mathrm{H} & -0.753981 & 1.985945 & 1.498160 \\ \mathrm{H} & -0.507332 & -1.963728 & -0.515576 \\ \mathrm{H} & -0.174306 & 1.168249 & -0.896582\end{array}$

\section{$T_{5-20}$}

21

$\begin{array}{lrrr}\mathrm{P} & -0.777028 & -0.464538 & -0.197917 \\ \mathrm{O} & 4.141599 & 0.155039 & -0.542582 \\ \mathrm{O} & 1.894259 & 0.136884 & -0.840399 \\ \mathrm{O} & -2.185936 & -1.303370 & 0.150538 \\ \mathrm{O} & -1.603070 & 1.089936 & -0.506123 \\ \mathrm{C} & 2.959648 & -0.017357 & -0.155422 \\ \mathrm{C} & 2.751704 & -0.453769 & 1.324985 \\ \mathrm{C} & -3.490965 & -0.747545 & -0.008608 \\ \mathrm{C} & -1.707907 & 2.034899 & 0.524351 \\ \mathrm{H} & 3.710713 & -0.678812 & 1.811527 \\ \mathrm{H} & 2.252312 & 0.356782 & 1.880591 \\ \mathrm{H} & 2.094156 & -1.335903 & 1.376760 \\ \mathrm{H} & -4.196070 & -1.569111 & 0.190137 \\ \mathrm{H} & -3.687332 & 0.067933 & 0.705515 \\ \mathrm{H} & -3.654753 & -0.362146 & -1.025210 \\ \mathrm{H} & -2.710240 & 2.035057 & 1.003450 \\ \mathrm{H} & -1.519327 & 3.044702 & 0.121725 \\ \mathrm{H} & -0.959464 & 1.847961 & 1.325663 \\ \mathrm{H} & -0.481352 & -0.420505 & -1.577748\end{array}$




$\begin{array}{rrrr}\mathrm{H} & -0.156291 & 0.167344 & 0.920698 \\ \mathrm{H} & -0.086640 & -1.710508 & 0.032347\end{array}$

\section{$T_{7-9 I n v}$}

16

$\begin{array}{llll}\mathrm{P} & -2.093633 & -0.748190 & -0.000003\end{array}$

$\begin{array}{llll}\mathrm{O} & 1.660292 & 1.205816 & -0.000037\end{array}$

$\begin{array}{llll}\mathrm{O} & 0.813932 & -0.907406 & -0.000032\end{array}$

$\begin{array}{llll}\text { O } & -2.242811 & 0.790614 & 0.000033\end{array}$

$\begin{array}{llll}\text { C } & 3.196623 & -0.628669 & 0.000031\end{array}$

$\begin{array}{llll}\text { C } & 1.771877 & -0.071686 & -0.000036\end{array}$

$\begin{array}{lrrr}\text { C } & -0.554060 & 1.424774 & 0.000012\end{array}$

$\begin{array}{llll}\mathrm{H} & 3.941714 & 0.174581 & -0.000695\end{array}$

$\begin{array}{llll}\mathrm{H} & 3.337287 & -1.265294 & 0.884798\end{array}$

$\begin{array}{llll}\mathrm{H} & 3.336902 & -1.266659 & -0.883804\end{array}$

H $\quad-3.395775 \quad-1.307956 \quad-0.000003$

$\mathrm{H} \quad-1.446361 \quad-1.305634 \quad-1.122481$

$\mathrm{H} \quad-1.446338 \quad-1.305686 \quad 1.122436$

$\begin{array}{llll}\mathrm{H} & -0.715605 & 2.502826 & 0.000032\end{array}$

$\begin{array}{llll}\mathrm{H} & -0.272648 & 0.969006 & -0.934738\end{array}$

$\begin{array}{llll}\mathrm{H} & -0.272614 & 0.968975 & 0.934738\end{array}$

\section{T $_{\text {7-9Ret }}$}

16

$\begin{array}{lrrr}\mathrm{P} & -1.545951 & -0.972764 & 0.109945 \\ \mathrm{O} & 1.447275 & 0.241796 & 1.332843 \\ \mathrm{O} & 0.486652 & 0.192850 & -0.709187 \\ \mathrm{O} & -2.122074 & 0.385236 & -0.277882 \\ \mathrm{C} & 2.742950 & -0.564510 & -0.541997 \\ \mathrm{C} & 1.485400 & -0.003483 & 0.118489 \\ \mathrm{C} & -0.630767 & 1.945870 & -0.077678 \\ \mathrm{H} & 3.413989 & -0.977594 & 0.218649 \\ \mathrm{H} & 3.258416 & 0.253463 & -1.066581 \\ \mathrm{H} & 2.491955 & -1.324950 & -1.291970 \\ \mathrm{H} & 0.125822 & 1.890105 & 0.703597 \\ \mathrm{H} & -2.611407 & -1.774042 & 0.611018\end{array}$




$\begin{array}{rrrr}\mathrm{H} & -1.615090 & 2.278673 & 0.234123 \\ \mathrm{H} & -1.023066 & -1.798962 & -0.911350 \\ \mathrm{H} & -0.609805 & -1.016203 & 1.171080 \\ \mathrm{H} & -0.321868 & 2.234649 & -1.076827\end{array}$




\section{Effect of Substitution on Phosphorus}

The transition states for inversion $\mathbf{T}_{\mathbf{7 X - 9}}$ and retention $\mathbf{T}_{\mathbf{2 1 X} \text {-9 }}$ (Chart S3) for different substituents on phosphorus have been calculated. Geometries were optimized at the BP86/6-311++G(3df,3pd) level of theory. A smaller basis set $[6-311+G(d, p)$ except for the phenyl substituents for which the 6-31G(d) basis was employed] was used for the calculations of the two transition structures of the $\mathrm{PPh}_{3}$ system. Solvent effects were incorporated by the C-PCM model using acetonitrile $(\varepsilon=36.64)$ as solvent.<smiles>[R7]P([R])O[SiH3]OC(C)=O</smiles>

$T_{7 X-9}$ inversion

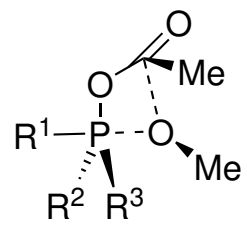

$T_{21 X-9}$ retention

\begin{tabular}{|c|c|c|c|}
\hline & $\mathrm{R}^{1}$ & $\mathrm{R}^{2}$ & $\mathrm{R}^{3}$ \\
\hline \multicolumn{4}{|c|}{ inversion } \\
\hline $\mathbf{T}_{7 \mathrm{~m} 1-9}$ & $\mathrm{Me}$ & $\mathrm{H}$ & $\mathrm{H}$ \\
\hline $\mathbf{T}_{7 \mathrm{~m} 2-9}$ & $\mathrm{Me}$ & $\mathrm{Me}$ & $\mathrm{H}$ \\
\hline $\mathbf{T}_{7 \mathbf{m} 3-9}$ & $\mathrm{Me}$ & $\mathrm{Me}$ & $\mathrm{Me}$ \\
\hline$T_{\text {t7p3-9 }}$ & $\mathrm{Ph}$ & $\mathrm{Ph}$ & $\mathrm{Ph}$ \\
\hline \multicolumn{4}{|c|}{ retention } \\
\hline $\mathbf{T}_{21 \mathrm{~m} 1-9}$ & $\mathrm{Me}$ & $\mathrm{H}$ & $\mathrm{H}$ \\
\hline$T_{21 \mathrm{~m} 2-9}$ & $\mathrm{Me}$ & $\mathrm{Me}$ & $\mathrm{H}$ \\
\hline$T_{21 \mathrm{~m} 3-9}$ & $\mathrm{Me}$ & $\mathrm{Me}$ & $\mathrm{Me}$ \\
\hline $\mathbf{T}_{21 \mathrm{p3-9}}$ & $\mathrm{Ph}$ & $\mathrm{Ph}$ & $\mathrm{Ph}$ \\
\hline
\end{tabular}

Chart S3: Compounds used in the investigation of stereochemical control

\subsection{Absolute Energies of Calculated Structures}

Table S6: Absolute energies in solution [a.u.]

\begin{tabular}{ccrrc}
\hline Compound & $E_{S C F}$ & $\Delta U$ & \multicolumn{1}{c}{$\Delta G$} & $N_{\text {imag }}$ \\
\hline $\mathbf{T}_{\mathbf{2 1 m 1 - 9}}$ & -726.259794757 & -726.114703 & -726.149781 & 1 \\
$\mathbf{T}_{\mathbf{2 1 m 2 - 9}}$ & -765.598984748 & -765.426404 & -765.463473 & 1 \\
$\mathbf{T}_{\mathbf{2 1 m 3 - 9}}$ & -804.930672338 & -804.730029 & -804.768753 & 1 \\
$\mathbf{T}_{\mathbf{2 1 p 3 - 9}}$ & -1380.02213901 & -1379.667383 & -1379.721320 & 1 \\
$\mathbf{T}_{7 \mathbf{7} 1-9}$ & -726.261076973 & -726.118401 & -726.157669 & 1 \\
$\mathbf{T}_{\mathbf{7 m 2 - 9}}$ & -765.607395596 & -765.437404 & -765.478521 & 1 \\
$\mathbf{T}_{7 \mathbf{7 m - 9}}$ & -804.947674154 & -804.749227 & -804.794129 & 1 \\
$\mathbf{T}_{\mathbf{7 p 3 - 9}}$ & -1380.04494601 & -1379.691780 & -1379.752441 & 1 \\
\hline
\end{tabular}




\subsection{Cartesian Coordinates of Calculated Structures}

All cartesian coordinates are given in Ångström. For convenience, coordinates of all compounds are also available in xyz-format packed together into a single ZIP-archive.

$\mathrm{T}_{21 \mathrm{~m} 1-9}$

19

$\begin{array}{llrr}\mathrm{P} & 1.261897 & 0.321767 & -0.115203\end{array}$

O $\quad 0.432844 \quad-1.039746 \quad-0.002893$

O $\quad-1.644393 \quad-1.323393 \quad-1.086159$

O $\quad-0.651651 \quad 0.905237 \quad-0.555294$

$\begin{array}{llll}\text { C } & 2.922465 & -0.345096 & 0.261522\end{array}$

C $\quad-1.694265-0.716355 \quad 1.255806$

$\begin{array}{llll}\text { C } & -1.241098 & 2.027619 & 0.087534\end{array}$

$\begin{array}{llll}\text { C } & -1.111921 & -0.766668 & -0.138994\end{array}$

H $\quad 3.642392 \quad 0.486593 \quad 0.225719$

H $\quad 3.212410 \quad-1.098949 \quad-0.482299$

$\begin{array}{llll}\mathrm{H} & 2.943658 & -0.796134 & 1.262181\end{array}$

H $\quad 1.526700 \quad 0.920803 \quad-1.369614$

$\begin{array}{llll}\mathrm{H} & 1.166877 & 1.303856 & 0.904697\end{array}$

$\begin{array}{llll}\mathrm{H} & -2.722256 & -0.334863 & 1.210327\end{array}$

$\begin{array}{llll}\mathrm{H} & -2.337979 & 1.934682 & 0.073197\end{array}$

$\begin{array}{llll}\mathrm{H} & -1.727358 & -1.746226 & 1.638828\end{array}$

$\mathrm{H} \quad-1.105536 \quad-0.106136 \quad 1.950060$

$\begin{array}{llll}\mathrm{H} & -0.963526 & 2.940684 & -0.462589\end{array}$

$\begin{array}{llll}\mathrm{H} & -0.909329 & 2.135405 & 1.137095\end{array}$

\section{$T_{21 m 2-9}$}

22

$\begin{array}{llll}\mathrm{P} & -1.103051 & 0.013363 & -0.227133\end{array}$

$\begin{array}{llll}\mathrm{O} & 1.841835 & -0.788161 & 1.484998\end{array}$

$\begin{array}{llll}\mathrm{O} & 0.647916 & 1.025132 & 0.200690\end{array}$

O $\quad-0.077916 \quad-1.151874 \quad 0.200462$

$\begin{array}{llll}\text { C } & 2.118410 & -0.885127 & -0.913878\end{array}$

$\begin{array}{llll}\text { C } & 1.357662 & -0.635097 & 0.371316\end{array}$

$\begin{array}{llll}\text { C } & 1.170311 & 1.974568 & -0.713428\end{array}$

$\begin{array}{llll}\text { C } & -2.499963 & -1.104204 & -0.642349\end{array}$

C $\quad-1.867675 \quad 1.099871 \quad 1.016029$ 


$\begin{array}{lrrr}\mathrm{H} & 3.076644 & -0.351572 & -0.885089 \\ \mathrm{H} & 2.328626 & -1.963032 & -0.970287 \\ \mathrm{H} & 2.268872 & 2.007916 & -0.638972 \\ \mathrm{H} & 1.561062 & -0.593196 & -1.811348 \\ \mathrm{H} & 0.899370 & 1.746031 & -1.762497 \\ \mathrm{H} & 0.780305 & 2.975452 & -0.465033 \\ \mathrm{H} & -3.338471 & -0.503374 & -1.023785 \\ \mathrm{H} & -2.958972 & 0.990189 & 1.006726 \\ \mathrm{H} & -2.834555 & -1.659183 & 0.244745 \\ \mathrm{H} & -2.192308 & -1.819530 & -1.416172 \\ \mathrm{H} & -1.580763 & 2.141137 & 0.834589 \\ \mathrm{H} & -1.478225 & 0.805654 & 2.000093 \\ \mathrm{H} & -0.952981 & 0.642224 & -1.501322\end{array}$

\section{T21m3-9}

25

$\begin{array}{lrrr}\mathrm{P} & 1.005997 & -0.092266 & 0.008614 \\ \mathrm{O} & -2.147855 & -1.133681 & -1.166372 \\ \mathrm{O} & -0.680155 & 0.901985 & -0.467008 \\ \mathrm{O} & -0.150682 & -1.247504 & -0.023949 \\ \mathrm{C} & 2.325924 & -1.361780 & 0.299114 \\ \mathrm{C} & 1.699902 & 0.670971 & -1.497654 \\ \mathrm{C} & 1.270531 & 0.901824 & 1.512806 \\ \mathrm{C} & -2.203624 & -0.557299 & 1.175505 \\ \mathrm{C} & -1.542372 & -0.765595 & -0.168689 \\ \mathrm{C} & -1.146495 & 2.176594 & -0.079785 \\ \mathrm{H} & 3.306989 & -0.875937 & 0.391991 \\ \mathrm{H} & 2.793819 & 0.581721 & -1.494890 \\ \mathrm{H} & 2.360993 & -2.065593 & -0.543572 \\ \mathrm{H} & 2.351720 & 1.062286 & 1.622820 \\ \mathrm{H} & 2.119399 & -1.928327 & 1.216781 \\ \mathrm{H} & 1.396235 & 1.718938 & -1.584824 \\ \mathrm{H} & 1.298587 & 0.122432 & -2.361152 \\ \mathrm{H} & 0.929001 & 0.316499 & 2.378042 \\ \mathrm{H} & 0.756565 & 1.866283 & 1.500065 \\ \mathrm{H} & -3.119724 & 0.031407 & 1.044372 \\ \mathrm{H} & -2.485436 & -1.544989 & 1.571150 \\ \mathrm{H} & -2.078635 & 2.402813 & -0.624339 \\ \mathrm{H} & -1.550909 & -0.066181 & 1.904842\end{array}$




$\begin{array}{rrrr}\mathrm{H} & -1.352936 & 2.251470 & 1.004346 \\ \mathrm{H} & -0.409279 & 2.956464 & -0.343985\end{array}$

$T_{21 p 3-9}$

46

$\begin{array}{llll}\mathrm{P} & 0.036426 & -0.221697 & 0.285093\end{array}$

$\begin{array}{llll}\text { O } & 0.270871 & -2.143333 & 0.357778\end{array}$

$\begin{array}{llll}\mathrm{O} & 0.186861 & -0.381115 & 1.961145\end{array}$

$\begin{array}{llll}\mathrm{O} & -0.176215 & -2.253999 & 3.195997\end{array}$

$\begin{array}{llll}\text { C } & 3.937375 & -0.101893 & -2.295111\end{array}$

$\begin{array}{llll}\text { C } & 3.678479 & 0.895190 & -1.339096\end{array}$

$\begin{array}{llll}\text { C } & 3.017409 & -1.148961 & -2.478138\end{array}$

$\begin{array}{llll}\text { C } & 2.491700 & 0.862379 & -0.588519\end{array}$

$\begin{array}{llll}\text { C } & 2.098831 & -1.843743 & 2.477411\end{array}$

$\begin{array}{llll}\text { C } & 1.849111 & -1.208301 & -1.701947\end{array}$

C $\quad \begin{array}{llll}\text { C } & 1.562923 & -0.190881 & -0.760663\end{array}$

$\begin{array}{llll}\text { C } & 0.605704 & -1.636199 & 2.494402\end{array}$

$\begin{array}{llll}\text { C } & -4.134763 & -0.786432 & -1.723908\end{array}$

$\begin{array}{llll}\text { C } & -4.024418 & -0.432306 & -0.367404\end{array}$

$\begin{array}{llll}\text { C } & -2.973535 & -0.971853 & -2.493734\end{array}$

$\begin{array}{llll}\text { C } & -2.759123 & -0.251973 & 0.215718\end{array}$

$\begin{array}{llll}\text { C } & -1.702982 & -0.817427 & -1.910850\end{array}$

$\begin{array}{llll}\text { C } & -1.591852 & -0.447426 & -0.554344\end{array}$

$\begin{array}{llll}\text { C } & -0.770344 & -3.111104 & 0.326690\end{array}$

$\begin{array}{llll}\text { C } & -0.662236 & 3.803286 & -0.598261\end{array}$

$\begin{array}{llll}\text { C } & -0.506319 & 4.478477 & 0.626176\end{array}$

$\begin{array}{llll}\text { C } & -0.499237 & 2.410507 & -0.659450\end{array}$

$\begin{array}{llll}\text { C } & -0.179346 & 3.748622 & 1.779652\end{array}$

$\begin{array}{llll}\text { C } & -0.169538 & 1.659724 & 0.494569\end{array}$

$\begin{array}{llll}\text { C } & -0.008120 & 2.352610 & 1.714279\end{array}$

$\begin{array}{llll}\mathrm{H} & 4.858774 & -0.066391 & -2.892459\end{array}$

$\begin{array}{llll}\mathrm{H} & 4.397459 & 1.710033 & -1.179147\end{array}$

$\begin{array}{llll}\mathrm{H} & 3.216112 & -1.936630 & -3.217581\end{array}$

$\mathrm{H} \quad 2.558246 \quad-1.474355 \quad 1.553366$

$\mathrm{H} \quad 2.524584 \quad-1.286301 \quad 3.331384$

$\begin{array}{llll}\mathrm{H} & 2.325007 & -2.909994 & 2.611999\end{array}$

$\begin{array}{llrr}\mathrm{H} & 2.296990 & 1.654766 & 0.141487\end{array}$

$\mathrm{H} \quad 1.168032 \quad-2.055978 \quad-1.810087$

$\begin{array}{llll}\mathrm{H} & 0.253367 & 1.800180 & 2.622873\end{array}$ 


$\begin{array}{lrrr}\mathrm{H} & -5.125104 & -0.915766 & -2.181048 \\ \mathrm{H} & -4.927293 & -0.285444 & 0.241065 \\ \mathrm{H} & -3.050816 & -1.243260 & -3.555479 \\ \mathrm{H} & -2.686736 & 0.045922 & 1.270889 \\ \mathrm{H} & -1.518748 & -2.961821 & 1.128990 \\ \mathrm{H} & -1.298123 & -3.120187 & -0.643785 \\ \mathrm{H} & -0.916237 & 4.362019 & -1.509674 \\ \mathrm{H} & -0.807645 & -0.976844 & -2.522914 \\ \mathrm{H} & -0.639573 & 5.567779 & 0.678065 \\ \mathrm{H} & -0.632455 & 1.906595 & -1.626007 \\ \mathrm{H} & -0.299704 & -4.099575 & 0.475744 \\ \mathrm{H} & -0.052977 & 4.264517 & 2.741721\end{array}$

\section{$\mathrm{T}_{7 \mathrm{~m} 1-9}$}

19

$\begin{array}{lrrr}\mathrm{P} & -2.767757 & -0.422343 & -0.003373 \\ \mathrm{O} & 2.786969 & 1.333006 & -0.005485 \\ \mathrm{O} & 2.116146 & -0.828976 & -0.006035 \\ \mathrm{O} & -1.589891 & 0.575924 & -0.002548 \\ \mathrm{C} & 4.468393 & -0.383212 & 0.010634 \\ \mathrm{C} & 3.011305 & 0.095331 & -0.004596 \\ \mathrm{C} & 0.098252 & -0.072256 & -0.004213 \\ \mathrm{C} & -4.352957 & 0.416621 & 0.012024 \\ \mathrm{H} & 5.029225 & 0.113536 & -0.793198 \\ \mathrm{H} & 4.933326 & -0.085943 & 0.962215 \\ \mathrm{H} & 4.546866 & -1.470787 & -0.101165 \\ \mathrm{H} & 0.571454 & 0.903198 & -0.002657 \\ \mathrm{H} & 0.046452 & -0.624732 & 0.927418 \\ \mathrm{H} & 0.046235 & -0.621265 & -0.937862 \\ \mathrm{H} & -5.149814 & -0.341806 & 0.010837 \\ \mathrm{H} & -4.440416 & 1.047127 & -0.882249 \\ \mathrm{H} & -4.430029 & 1.033487 & 0.916694 \\ \mathrm{H} & -2.752962 & -1.289770 & -1.131545 \\ \mathrm{H} & -2.739726 & -1.306429 & 1.111559\end{array}$




\section{$T_{7 m 2-9}$}

22

$\begin{array}{lrrr}\mathrm{P} & -2.478534 & 0.023388 & 0.276969 \\ \mathrm{O} & 3.157760 & -1.243405 & -0.471966 \\ \mathrm{O} & 2.388024 & 0.759546 & 0.250297 \\ \mathrm{O} & -1.257354 & -0.629312 & -0.411054 \\ \mathrm{C} & 4.752539 & 0.393711 & 0.271571 \\ \mathrm{C} & 3.324123 & -0.083695 & -0.016940 \\ \mathrm{C} & 0.425186 & 0.015959 & -0.102087 \\ \mathrm{C} & -3.880524 & -1.104717 & 0.240184 \\ \mathrm{C} & -2.976313 & 1.591843 & -0.461702 \\ \mathrm{H} & 5.398336 & 0.179271 & -0.590781 \\ \mathrm{H} & 5.151109 & -0.173136 & 1.126173 \\ \mathrm{H} & 4.789718 & 1.463479 & 0.507687 \\ \mathrm{H} & 0.917779 & -0.891313 & -0.431057 \\ \mathrm{H} & 0.365512 & 0.855883 & -0.782758 \\ \mathrm{H} & 0.316146 & 0.194132 & 0.962173 \\ \mathrm{H} & -4.730576 & -0.650168 & 0.768388 \\ \mathrm{H} & -4.159764 & -1.305721 & -0.802924 \\ \mathrm{H} & -3.837200 & 1.998342 & 0.088225 \\ \mathrm{H} & -3.600205 & -2.043455 & 0.734890 \\ \mathrm{H} & -3.252223 & 1.430366 & -1.512744 \\ \mathrm{H} & -2.212505 & 0.310403 & 1.660714 \\ \mathrm{H} & -2.145622 & 2.307858 & -0.406886\end{array}$

\section{$T_{7 m 3-9}$}

25

$\begin{array}{lrrr}\mathrm{P} & -2.315748 & 0.028175 & 0.000928 \\ \mathrm{O} & 3.414676 & 1.349508 & -0.002304 \\ \mathrm{O} & 2.602560 & -0.764117 & -0.010226 \\ \mathrm{O} & -1.006872 & 0.853003 & -0.007164 \\ \mathrm{C} & 4.977806 & -0.475144 & 0.009429 \\ \mathrm{C} & 3.557174 & 0.101084 & -0.004901 \\ \mathrm{C} & 0.664727 & 0.088539 & -0.008040 \\ \mathrm{C} & -3.705624 & 1.178483 & 0.008361 \\ \mathrm{C} & -2.479405 & -1.033447 & -1.453502 \\ \mathrm{C} & -2.462990 & -1.031535 & 1.458507\end{array}$




$\begin{array}{rrrr}\mathrm{H} & 5.567875 & -0.021693 & -0.799041 \\ \mathrm{H} & 5.464798 & -0.204556 & 0.957840 \\ \mathrm{H} & 4.982266 & -1.566040 & -0.096911 \\ \mathrm{H} & 1.170161 & 1.046629 & -0.008938 \\ \mathrm{H} & 0.567350 & -0.449907 & 0.926169 \\ \mathrm{H} & 0.565165 & -0.451085 & -0.941328 \\ \mathrm{H} & -4.654618 & 0.624425 & 0.015201 \\ \mathrm{H} & -3.656247 & 1.809876 & -0.888642 \\ \mathrm{H} & -3.644533 & 1.812065 & 0.903092 \\ \mathrm{H} & -3.450882 & -1.546900 & -1.436237 \\ \mathrm{H} & -3.435129 & -1.544006 & 1.453224 \\ \mathrm{H} & -2.408089 & -0.418596 & -2.360746 \\ \mathrm{H} & -2.380526 & -0.415617 & 2.364089 \\ \mathrm{H} & -1.679981 & -1.786604 & -1.461126 \\ \mathrm{H} & -1.664431 & -1.785643 & 1.457867\end{array}$

\section{$T_{7 p 3-9}$}

$\begin{array}{lrrr}46 & & & \\ \mathrm{P} & 0.634191 & -0.016702 & -0.230511 \\ \mathrm{O} & -4.855023 & -0.689370 & -2.282388 \\ \mathrm{O} & -4.324402 & 0.261257 & -0.290936 \\ \mathrm{O} & -0.513271 & -0.206369 & -1.286568 \\ \mathrm{C} & 4.662599 & 0.029098 & -2.505032 \\ \mathrm{C} & 4.590700 & 0.573093 & -1.209997 \\ \mathrm{C} & 3.510739 & -0.497810 & -3.115221 \\ \mathrm{C} & 3.368436 & 0.592857 & -0.519768 \\ \mathrm{C} & 2.283234 & -0.485901 & -2.433558 \\ \mathrm{C} & 2.210578 & 0.064029 & -1.134437 \\ \mathrm{C} & 1.517855 & -2.577114 & 2.892303 \\ \mathrm{C} & 1.457642 & -1.428569 & 2.086994 \\ \mathrm{C} & 0.818382 & 2.742794 & 0.168591 \\ \mathrm{C} & 0.815612 & -3.738975 & 2.523277 \\ \mathrm{C} & 0.684275 & -1.444300 & 0.903012 \\ \mathrm{C} & 0.565775 & 3.940269 & 0.854596 \\ \mathrm{C} & 0.432979 & 1.506797 & 0.741745 \\ \mathrm{C} & 0.054313 & -3.758509 & 1.342189 \\ \mathrm{C} & -6.643281 & -0.069970 & -0.789392 \\ \mathrm{C} & -5.160850 & -0.176927 & -1.172751 \\ \mathrm{C} & -2.289411 & 0.008623 & -0.804481\end{array}$




$\begin{array}{lrrr}\mathrm{C} & -0.473398 & 2.690765 & 2.672185 \\ \mathrm{C} & -0.226283 & 1.486763 & 1.993075 \\ \mathrm{C} & -0.074748 & 3.914675 & 2.106720 \\ \mathrm{C} & -0.010882 & -2.615958 & 0.527868 \\ \mathrm{H} & 5.621210 & 0.018186 & -3.041425 \\ \mathrm{H} & 5.488521 & 0.990449 & -0.734583 \\ \mathrm{H} & 3.565134 & -0.919760 & -4.127811 \\ \mathrm{H} & 3.323450 & 1.029414 & 0.487040 \\ \mathrm{H} & 2.115318 & -2.561962 & 3.813782 \\ \mathrm{H} & 2.008860 & -0.527779 & 2.390165 \\ \mathrm{H} & 1.382425 & -0.894380 & -2.910032 \\ \mathrm{H} & 1.314836 & 2.774431 & -0.810460 \\ \mathrm{H} & 0.872977 & 4.896165 & 0.409568 \\ \mathrm{H} & 0.865554 & -4.633412 & 3.159075 \\ \mathrm{H} & -7.236845 & 0.252738 & -1.658140 \\ \mathrm{H} & -7.005844 & -1.069310 & -0.494522 \\ \mathrm{H} & -6.800909 & 0.619985 & 0.051271 \\ \mathrm{H} & -2.647180 & -0.418073 & -1.736634 \\ \mathrm{H} & -2.277925 & -0.610921 & 0.086861 \\ \mathrm{H} & -2.241738 & 1.086827 & -0.694473 \\ \mathrm{H} & -0.980098 & 2.669734 & 3.646317 \\ \mathrm{H} & -0.595460 & -2.637305 & -0.400185 \\ \mathrm{H} & -0.549139 & 0.538159 & 2.440361 \\ \mathrm{H} & -0.492523 & -4.665213 & 1.050358 \\ \mathrm{H} & -0.267524 & 4.854035 & 2.642750\end{array}$

